\title{
Laser Theory for Optomechanics: Limit Cycles in the Quantum Regime
}

\author{
Niels Lörch, ${ }^{1,2}$ Jiang Qian, ${ }^{3}$ Aashish Clerk, ${ }^{4}$ Florian Marquardt, ${ }^{5,6}$ and Klemens Hammerer ${ }^{1,2}$ \\ ${ }^{1}$ Institut für Gravitationsphysik, Leibniz Universität Hannover \\ and Max-Planck-Institut für Gravitationsphysik (Albert-Einstein-Institut), \\ Callinstraße 38, 30167 Hannover, Germany \\ ${ }^{2}$ Institut für Theoretische Physik, Leibniz Universität Hannover, \\ Appelstraße 2, 30167 Hannover, Germany \\ ${ }^{3}$ Arnold Sommerfeld Center for Theoretical Physics, Center for NanoScience and Department of Physics, \\ Ludwig-Maximilians-Universität München, Theresienstrasse 37, 80333 München, Germany \\ ${ }^{4}$ Department of Physics, McGill University, Montreal, Quebec H3A 2T8, Canada \\ ${ }^{5}$ Friedrich-Alexander-Universität Erlangen-Nürnberg, Staudtstraße 7, D-91058 Erlangen, Germany \\ ${ }^{6}$ Max Planck Institute for the Science of Light, \\ Günther-Scharowsky-Straße 1/Bau 24, D-91058 Erlangen, Germany
}

(Received 4 October 2013; published 31 January 2014; publisher error corrected 11 February 2014)

\begin{abstract}
Optomechanical systems can exhibit self-sustained limit cycles where the quantum state of the mechanical resonator possesses nonclassical characteristics such as a strongly negative Wigner density, as was shown recently in a numerical study by Qian et al. [Phys. Rev. Lett. 109, 253601 (2012)]. Here, we derive a Fokker-Planck equation describing mechanical limit cycles in the quantum regime that correctly reproduces the numerically observed nonclassical features. The derivation starts from the standard optomechanical master equation and is based on techniques borrowed from the laser theory due to Haake and Lewenstein. We compare our analytical model with numerical solutions of the master equation based on Monte Carlo simulations and find very good agreement over a wide and so far unexplored regime of system parameters. As one main conclusion, we predict negative Wigner functions to be observable even for surprisingly classical parameters, i.e., outside the single-photon strong-coupling regime, for strong cavity drive and rather large limit-cycle amplitudes. The approach taken here provides a natural starting point for further studies of quantum effects in optomechanics.
\end{abstract}

DOI: 10.1103/PhysRevX.4.011015

Subject Areas: Nanophysics, Quantum Physics

\section{INTRODUCTION}

Optomechanical systems provide a test bed to study a broad range of paradigmatic quantum optical processes at so far unexplored meso- and macroscopic mass and length scales [1-3]. That quantum effects can play an important and even dominating role in the dynamics of these systems has been shown in a number of recent experiments demonstrating cooling to the quantum ground state $[4,5]$, ponderomotive squeezing of light [6,7], backaction noiselimited position sensing [8,9], coherent state transfer [10], and entanglement [11].

In the most elementary optomechanical setup, a single cavity mode couples to a single mechanical oscillator through, e.g., radiation-pressure or dipole-gradient forces. The dynamics of the system depends crucially on the frequency of the external driving field applied to the cavity: For the purpose of position or force sensing, as in Refs. [8,9], the driving field is chosen resonant, while for backaction

Published by the American Physical Society under the terms of the Creative Commons Attribution 3.0 License. Further distribution of this work must maintain attribution to the author(s) and the published article's title, journal citation, and DOI. cooling or state transfer, the field is tuned below (to the red side of) the cavity frequency $[4,5,10]$. For blue detuning, the system exhibits a rather complex nonlinear behavior. When the driving field is swept from the red to the blue side, the nonlinear dynamics sets in as a parametric amplification process where phonons and photons are created as correlated in pairs [12]. This correlation lies at the heart of the recently reported generation of optomechanical entanglement [11]. The amplification will finally go over into a regime of self-sustained limit cycles due to the nonlinearity inherent to the optomechanical coupling. The classical dynamics in this regime has been observed experimentally [13-18] and is well studied theoretically [19-22]. Motivated by the impressive progress toward quantum effects in optomechanical systems, the quantum regime of optomechanical limit cycles also received significant attention in theoretical studies [23-29].

In particular, a recent numerical study of the full optomechanical master equation in the limit-cycle regime showed that the Wigner function of the mechanical oscillator can become strongly negative [27]: Negativities of the Wigner function occur for driving fields at the blue sidebands and-more pronouncedly-also occur for resonant drive. Limit-cycle states with negative Wigner density even 
exist in regions of red detuning where a (simple) classical model would not predict limit cycles at all. The numerical findings were independently confirmed in Ref. [28]. This reference predicts negative Wigner density even on higher sidebands and compares the extent of negativity found for different detunings in more detail. In view of these findings, it is important to strive for a deeper understanding of these effects and the underlying mechanisms on the basis of an appropriate analytical model.

The transition from parametric amplification to optomechanical limit cycles can be understood in analogy to the threshold behavior of a laser (or maser) cavity [30-32] where the roles of the laser cavity and the laser medium are played by, respectively, the mechanical oscillator and the optomechanical cavity [33]. Along this line, a semiclassical rate-equation model was derived in Refs. [21,33] for optomechanical systems. Rodrigues and Armour [25,26] developed a quantum mechanical treatment employing a truncated Wigner-function approach to derive a FokkerPlanck equation (FPE) for the mechanical oscillator. The FPE predicted, in particular, a sub-Poissonian, or numbersqueezed, phonon statistics in the limit cycle when the driving field is blue detuned from the cavity resonance by the mechanical oscillation frequency.

In the present article, we apply the laser theory due to Haake and Lewenstein [31,32] to describe optomechanical limit cycles in the quantum regime. Our model correctly reproduces the characteristics of limit cycles mentioned above. It identifies general requirements on system parameters (such as coupling strength, driving power, sideband resolution, temperature, etc.) for the occurrence of sub-Poissonian phonon statistics and negative Wigner functions, and establishes a tight connection between the two phenomena. We find that negative Wigner functions can also be achieved in rather classical parameter regimes where the coupling per single photon $g_{0}$ is smaller than the cavity linewidth, and where the cavity is driven strongly and limit-cycle amplitudes are large. The associated small Fano factors are lower bounded by, and can reach, the sideband parameter $\kappa / \omega_{m}$ (ratio of cavity linewidth to mechanical resonance frequency) for sufficiently strong optomechanical cooperativity.

Starting from the standard optomechanical master equation [1,2], an effective FPE is derived for the quasiprobability distribution (such as, e.g., the Wigner, $P$, or $Q$ function) of the mechanical oscillator under adiabatic elimination of the cavity mode. The nonlinearity of the optomechanical interaction gives rise to nonlinear drift and diffusion coefficients in the FPE that describe, respectively, the (classical) nonlinear physics of limit cycles $[19,20]$ and the impact of quantum noise of the cavity. The approach taken here permits us to work in a picture that interpolates between the dressed-state picture introduced in Refs. [34,35] through a polaron transformation and the bare-state picture of the standard master equation $[1,2,25,28,36]$. Remarkably, in analogy to the polaron picture, this intermediate picture explicitly separates the optical Kerr nonlinearity inherent to the radiation pressure from the optomechanical interaction. In contrast to the polaron picture, the interaction term is not removed from the master equation, and both the mechanical oscillator and the cavity remain separate systems, as in the standard master-equation picture. The entanglement of cavity and oscillator in the polaron picture would complicate the study of them as separate systems, as required in the context of limit cycles. As we will show, the novel treatment of the optomechanical Kerr nonlinearity presented in this article can become essential to understanding the physics of limit cycles.

The effective FPE derived here exactly reproduces the one of Rodrigues and Armour $[25,26]$ when neglecting the different description of the Kerr nonlinearity of the cavity, which is treated in the standard master-equation picture there. In comparison to Refs. [25,26], our approach does not require truncation of higher-order derivatives and gives a consistent and natural account of the Kerr nonlinearity.

The article is organized as follows: In Sec. II, we give an executive summary of the main results, as far as they relate to the appearance of nonclassical mechanical states. In Sec. III, we introduce the main idea of Haake and Lewenstein's laser theory in the context of optomechanics and apply it to derive the effective FPE for the mechanical oscillator. In Sec. IV, we discuss the implications of the FPE equation for optomechanical limit cycles in the quantum regime. In principle, each of these sections can be read independently. Readers who are interested only in one particular aspect are encouraged to jump directly to the respective section of interest.

\section{PREVIEW OF THE MAIN RESULTS}

The aim of this section is to give a preview of our most important results and to indicate how these results could be derived in a relatively simple (quantum-noise) approach. The main idea is to find the width of the mechanical limit cycles in phase space and to deduce from that the spread in phonon numbers. For simplicity, we will assume here that the optomechanical interaction dominates (i.e., formally zero mechanical damping). The full optomechanical lasertheory analysis will go significantly beyond this simplified model, but it will reproduce the features discussed here.

In the following, we will find it convenient to characterize the optomechanical coupling in several ways: as the cavity-frequency shift per displacement $G$, via the singlephoton coupling strength $g_{0}=G x_{\mathrm{ZPF}}$, and via the dimensionless ratio $\eta=2 g_{0} / \omega_{m} . x_{\mathrm{ZPF}}=\sqrt{\hbar / 2 m \omega_{m}}$ is the zero-point amplitude of the mechanical oscillator with mass $m$ and frequency $\omega_{m}$. We start by assuming mechanical oscillations at a fixed amplitude $r$ such that $x(t)=$ $x_{\mathrm{ZPF}} \operatorname{Re}\left[r e^{-i \omega_{m} t}\right]$. At each instant of time, the radiationpressure force $F=\hbar G a^{\dagger} a$ ( $a$ is the photon-annihilation operator) will feed energy into the mechanical oscillations at a rate (power) $P=F(t) \dot{x}(t)$. Following the classical 
approach [19], we can predict the slow drift of the mechanical oscillation amplitude by calculating the average power input $\overline{\langle P(t)\rangle}$. Here, the angled brackets denote the quantum expectation value, while the overbar averages over a time window comprising several oscillation periods. We note that the power-balance equation is analogous to the loss-gain equations in a laser and that the laser analogy will be heavily used throughout the manuscript.

In addition to this drift, however, there will be diffusion of the mechanical oscillator's energy, due to the fundamental radiation-pressure shot-noise fluctuations. The energy-diffusion constant is given by $D_{E}=$ $\frac{1}{2} \int_{-\infty}^{+\infty} d \tau \overline{\langle\delta P(t+\tau) \delta P(t)\rangle}$, where $\delta P(t)=P(t)-\langle P(t)\rangle$ denotes the fluctuations. In order to discuss the quantum dynamics of optomechanical limit cycles, it turns out to be crucial to study the behavior of this diffusion constant as a function of cycle amplitude. In particular, we will show that the appearance of nonclassical mechanical states can only be understood by a rather subtle cancellation of some term that would usually dominate, leaving the diffusion constant small and leading to a narrowing of the phonon distribution by the sideband ratio $\kappa / \omega_{m}$ (where $\kappa$ denotes the cavityamplitude decay rate).

Our task of calculating this diffusion constant is complicated by the fact that we are dealing with shot noise inside an optical cavity whose resonance frequency oscillates sinusoidally. We thus have to solve the equation for the light field inside such a mechanically driven cavity, i.e., $d a / d t=\{-i[\Delta-G x(t)]-\kappa\} a+\sqrt{2 \kappa} a_{\text {in }}(t)$, where $\Delta=$ $\omega_{L}-\omega_{c}$ is the detuning between the laser at frequency $\omega_{L}$ and the bare cavity resonance at $\omega_{c}$. The solution for $a(t)$ can be expressed via the extra phase $\theta(t)=\eta \operatorname{Im}\left[r e^{-i \omega_{m} t}\right]$ accumulated in the cavity field due to the oscillations. It reads

$$
a(t)=e^{-i \theta(t)} \int_{-\infty}^{t} d t^{\prime} \chi_{c}\left(t-t^{\prime}\right) e^{i \theta\left(t^{\prime}\right)} a_{\mathrm{in}}\left(t^{\prime}\right),
$$

where $\chi_{c}(t)=\sqrt{2 \kappa} \exp [(i \Delta-\kappa) t]$ is the standard cavity filter function (and $\theta=0$ recovers the usual case).

The light intensity oscillates at harmonics of the mechanical motion. $\alpha(t)=e^{i \theta(t)}\langle a(t)\rangle=\sum_{n} \alpha_{n} e^{i n \omega_{m} t}$ is the average cavity amplitude (modulo the phase) that can be obtained by evaluating Eq. (1). For a constant laser drive, with an amplitude $\sqrt{2 \kappa}\left\langle a_{\text {in }}\right\rangle \equiv E$, we obtain $\alpha_{n}=$ $E e^{-i n \phi} J_{-n}(\eta r) / h_{n}$, where $h_{n}=\kappa+i\left(n \omega_{m}-\Delta\right)$. These $\alpha_{n}$ are the Bessel amplitudes that also determine the appearance of multiple stable attractors in the classical analysis of the optomechanical instability [19]. These attractors can be found by noting that the drift of the amplitude $r$ is governed by the power input $\langle\overline{P(t)}\rangle$, as the energy of the mechanical oscillator is given by $m \omega_{m}^{2} r^{2} / 2$. In the regime of interest here, this drift can be approximated as

$\dot{r} \equiv \mu(r)=\frac{\langle P \overline{(t)}\rangle}{m \omega_{m}^{2} r} \simeq \frac{2 \kappa g_{0} E^{2}}{\omega_{m}^{2}} \frac{\Delta}{\Delta^{2}+\kappa^{2}} J_{0}(\eta r) J_{1}(\eta r)$.
The limit-cycle amplitude is thus fixed at the zeroes of the Bessel function, in the absence of additional mechanical damping. This observation will be crucial further below.

In addition, there are the electromagnetic vacuum fluctuations $\delta a_{\text {in }}(t)=a_{\text {in }}(t)-\left\langle a_{\text {in }}\right\rangle$ entering the cavity. In order to evaluate the mechanical energy-diffusion constant that is governed by those fluctuations, we need the force-force correlator $\left\langle F(t) F\left(t^{\prime}\right)\right\rangle$, i.e., ultimately the shotnoise (irreducible) part of the photon-number correlator. By using the vacuum-noise correlator $\left\langle\delta a_{\text {in }}(t) \delta a_{\text {in }}^{\dagger}(0)\right\rangle=\delta(t)$, we find directly

$$
\left\langle a^{\dagger}(t) a(t) a^{\dagger}\left(t^{\prime}\right) a\left(t^{\prime}\right)\right\rangle_{\mathrm{SN}}=e^{i \Delta\left(t-t^{\prime}\right)-\kappa\left|t-t^{\prime}\right|} \alpha^{*}(t) \alpha\left(t^{\prime}\right) .
$$

For a constant $\alpha$, this equation reduces to the shot-noise correlator employed in the quantum-noise approach to optomechanical cooling [37]. Now, we can proceed to evaluate the energy-diffusion constant $D_{E}$ introduced above. The resulting slightly lengthy expression [Eq. (B20) in Appendix B] can be simplified in the regime of interest here to

$D_{W} \equiv \frac{D_{E}}{\left(2 \hbar \omega_{m} r\right)^{2}} \simeq \kappa \frac{g_{0}^{2} E^{2}}{\omega_{m}^{4}}\left\{\frac{1}{2} \frac{\omega_{m}^{2}}{\kappa^{2}+\Delta^{2}} J_{0}^{2}(\eta r)+J_{1}^{2}(\eta r)\right\}$.

Here, we have introduced $D_{W}$ as the diffusion constant for the amplitude $r$ of the limit cycle. This amplitude is connected to the energy via $E=\hbar \omega_{m} r^{2}$, such that one obtains the relation between $D_{E}$ and $D_{W}$ shown here. It is now crucial to observe that the diffusion constant has a minimum right at the first limit cycle. This is because the first contribution in Eq. (3), which dominates at smaller amplitudes, is completely suppressed at the limit cycle, where $J_{0}(\eta r)=0$; see Eq. (2). Thus, only the second term survives, which is suppressed by a factor $\kappa^{2} / \omega_{m}^{2}$, i.e., the sideband ratio squared. We show in the main text that this suppression is caused by squeezing terms that exactly cancel the corresponding incoherent diffusion terms in leading order.

Now, we can combine these results to discuss the width $\sigma^{2}$ of the distribution in the amplitude $r$. The compromise between the diffusion at rate $D_{W}$ and the restoring force that drives $r$ back to the limit cycle results in a width $\sigma^{2}=-D_{W} / \mu^{\prime}$. For a fixed limit-cycle amplitude, both diffusion and drift scale as $g_{0}^{2} E^{2}$, such that the laser power and the optomechanical coupling drop out of this expression. This will change in the presence of mechanical damping and thermal fluctuations, but it still correctly describes the behavior once the optomechanical damping rate overwhelms the thermal fluctuations.

In order to estimate when the limit cycle may turn into a nonclassical mechanical quantum state, we will now look at the variance of the phonon number $\operatorname{Var}(n)$. Since $r$ is already measured in terms of the zero-point amplitude $x_{\mathrm{ZPF}}$, we have $r^{2}=n$. Thus, $\operatorname{Var}(n)=4\langle n\rangle \sigma^{2}$. This expression can be minimized by choosing an optimal detuning $(\Delta=\kappa)$, where we find $\operatorname{Var}(n)=\langle n\rangle\left(\kappa / \omega_{m}\right)$. In other 
words, in the resolved-sideband regime $\left(\kappa \ll \omega_{m}\right)$, one can get close to a mechanical Fock state $\operatorname{Var}(n)<1$, as long as the limit cycle is sufficiently small $\langle n\rangle<\omega_{m} / \kappa$. Note that the optomechanical-coupling strength $g_{0}$ enters indirectly here, since (in the absence of mechanical damping) the limit-cycle amplitude is determined by $J_{0}(\eta r)=0$, with $\eta=2 g_{0} / \omega_{m}$. Taking this observation into account, $\operatorname{Var}(n)<1$ is equivalent to $g_{0}^{2} / \omega_{m} \kappa>1$.4. However, it turns out that it is easier to produce a nonclassical state, i.e., one where the Wigner density has negative components. For the type of states relevant here, we numerically find that it is sufficient to have $\operatorname{Var}(n)<0.6\langle n\rangle^{0.7}$ for this purpose. Thus, the condition for nonclassicality reads approximately

$$
\frac{g_{0}}{\kappa}>2\left(\frac{\kappa}{\omega_{m}}\right)^{0.7},
$$

which is less stringent than the condition for achieving a Fock state, since one could still admit $g_{0} / \kappa<1$ if the sideband ratio $\omega_{m} / \kappa$ is sufficiently large.

In the simplified description given here, we have neglected several factors that will be discussed in our full analysis. This includes the effects of the mechanical damping, which will decrease the limit-cycle amplitude (shifting away from the point of the minimum diffusion constant). In addition, thermal fluctuations will add to the diffusion. Nevertheless, this effect can be overcome if the scale of the optically induced damping rate $\gamma_{\mathrm{opt}} \simeq \mu^{\prime}(r) \propto$ $g_{0}^{2} E^{2} / \omega_{m}^{3}$ dominates the influx of thermal phonons

$$
\left|\gamma_{\text {opt }}\right| \gg \gamma \bar{n},
$$

where $\bar{n}$ is the thermal phonon number of the bath and $\gamma$ is the mechanical damping rate. This condition is equivalent to the condition for ground-state cooling but here applied for the instable regime. It does not involve the coupling per single photon $g_{0}$ but only the linearized coupling $g \propto g_{0} E$, such that Eq. (5) essentially represents a condition on the strength of the driving field.

Another important aspect neglected here is the shift of the cavity resonance by the Kerr effect. This shift leads to an effective detuning $\Delta_{\text {eff }}$ that will enter all expressions instead of $\Delta$. The impact of this change is especially large near $\Delta \approx 0$, which is precisely the regime that we find to be optimal for nonclassical states.

The heuristic reasoning applied here and the resulting conditions (4) and (5) for achieving nonclassical mechanical states will receive a rigorous justification in Sec. IVon the basis of the Fokker-Planck equation derived in the next section.

\section{LASER THEORY FOR OPTOMECHANICS}

\section{A. Haake-Lewenstein laser-theory ansatz in optomechanics}

Master equation.- The standard master equation of an optomechanical system is $[1,2]$

$$
\frac{\mathrm{d}}{\mathrm{d} t} \rho=\left(L_{m}+L_{c}+L_{\text {int }}\right) \rho,
$$

where

$$
\begin{gathered}
L_{m} \rho=-i\left[\omega_{m} b^{\dagger} b, \rho\right]+\gamma(\bar{n}+1) D[b] \rho+\gamma \bar{n} D\left[b^{\dagger}\right] \rho, \\
L_{c} \rho=-i\left[-\Delta a^{\dagger} a-i E\left(a-a^{\dagger}\right), \rho\right]+\kappa D[a] \rho, \\
L_{\text {int }} \rho=-i\left[-g_{0} a^{\dagger} a\left(b+b^{\dagger}\right), \rho\right] .
\end{gathered}
$$

The three Liouvillians $L_{m}, L_{c}$, and $L_{\text {int }}$ refer to the mechanical oscillator, the cavity, and their interaction, respectively. $a$ and $b$ denote the annihilation operators of the cavity and the mechanical oscillator. The frequency of the mechanical oscillator is $\omega_{m}$, its amplitude damping rate is $\gamma=\omega_{m} / Q_{m}$, and its mean phonon number in thermal equilibrium is $\bar{n}$. We use the notation $D[A] \rho=2 A \rho A^{\dagger}-$ $A^{\dagger} A \rho-\rho A^{\dagger} A$ for Lindblad operators. $\kappa$ is the cavityamplitude decay rate, and $\Delta=\omega_{L}-\omega_{c}$ is the detuning from cavity resonance at $\omega_{c}$ of the driving field $E=$ $\sqrt{2 \kappa P_{L} / \hbar \omega_{L}}$ with power $P_{L}$ and frequency $\omega_{L}$. The master equation is written in a frame rotating at the frequency $\omega_{L}$ of the driving field. The optomechanical coupling per single photon is denoted by $g_{0}$ and essentially determines the dispersive shift of the cavity frequency with the displacement of the oscillator in units of the mechanical zero-point amplitude [38].

Note that in contrast to, e.g., Refs. [19,23,27], the definitions of $\gamma$ and $\kappa$ used here refer to the decay rate of the amplitude and will be used for all analytical results, in order to make the equations more readable. The corresponding decay rates for the energy $\kappa_{E}=2 \kappa$ and $\gamma_{E}=2 \gamma$ are the standard convention from Ref. [1]. For comparison to most experimental and numerical studies, we also provide the energy decay rates in the numerical results.

Our primary aim is to derive an effective equation of motion for the mirror based on the assumption that the dynamics of the cavity adiabatically follows the mechanical oscillator. This assumption will strictly be valid when the cavity decay rate $\kappa$ is larger than the characteristic coupling strength of the oscillator and the cavity mode (i.e., $g_{0}$ or the linear coupling $g=g_{0} \alpha$ enhanced by the mean cavity field $\alpha$ at the position of the limit cycle). As we will see, the resulting effective equation of motion for the mechanical oscillator also gives good results for the stationary state when this condition is barely fulfilled, and even when it is mildly violated.

Quasiprobability distribution.-Most importantly, we will not assume the usual linearization of the optomechanical coupling when we perform the adiabatic elimination. This is achieved by means of an ansatz inspired by laser theory $[31,32]$ that allows us to use a different adiabatic reference state of the cavity field for each point in the phase 
space of the mechanical oscillator. The idea is to switch to a phase-space representation for the mechanical degree of freedom. In principle, any quasiprobability distribution (e.g., $P$ distribution, Wigner function, etc.) can be used, but we will in the following mostly focus on the (Husimi) $Q$ function that yields the simplest formulas for the calculation presented below. In this formalism, the density operator $\rho$ is replaced by

$$
\sigma\left(\beta, \beta^{*}\right)=\frac{1}{\pi}\langle\beta|\rho| \beta\rangle,
$$

where $|\beta\rangle$ is a coherent state of the mechanical oscillator. In the Appendixes $\mathrm{A}$ and $\mathrm{B}$, we provide an extension and comparison of the present approach based on the $Q$ function to a general ( $s$-parametrized) quasiprobability distribution, including the $P$ distribution and Wigner function as special cases. $\sigma\left(\beta, \beta^{*}\right)$ is a density operator for the cavity field and a quasiprobability distribution for the oscillator over the complex phase-space variables $\left(\beta, \beta^{*}\right)$. The reduced density operator for the cavity is obtained by integrating over phase space

$$
\rho_{c}=\operatorname{tr}_{m}\{\rho\}=\int d^{2} \beta \sigma\left(\beta, \beta^{*}\right),
$$

and the quasiprobability distribution ( $Q$ function) for the oscillator follows on taking the trace over the cavity

$$
Q\left(\beta, \beta^{*}\right)=\operatorname{tr}_{c}\left\{\sigma\left(\beta, \beta^{*}\right)\right\} .
$$

$\sigma\left(\beta, \beta^{*}\right)$ itself still contains all information about the state of both systems and is fully equivalent to the density operator $\rho$. For the $Q$ function, the replacement rules [32]

$$
b^{\dagger} \rho \rightarrow \beta^{*} \sigma\left(\beta, \beta^{*}\right), \quad b \rho \rightarrow\left(\beta+\partial_{\beta^{*}}\right) \sigma\left(\beta, \beta^{*}\right)
$$

and their adjoints can be applied to the master equation (6) in order to arrive at an equivalent description in phase space of the oscillator. We use the notation $\partial_{\beta}$ to denote the partial derivative with respect to a variable $\beta$. The translated equation of motion is

$$
\partial_{t} \sigma\left(\beta, \beta^{*}, t\right)=\left(L_{m}+L_{c}+L_{\mathrm{int}}\right) \sigma\left(\beta, \beta^{*}, t\right),
$$

with

$$
\begin{aligned}
L_{m} \sigma= & {\left[\partial_{\beta}\left(\gamma-i \omega_{m}\right) \beta+\text { c.c. }\right] \sigma+2 \gamma(\bar{n}+1) \partial_{\beta \beta^{*}}^{2} \sigma, } \\
L_{c} \sigma= & L_{c} \sigma-i\left[-g_{0}\left(\beta+\beta^{*}\right) a^{\dagger} a, \sigma\right] \\
= & -i\left\{-\left[\Delta+2 g_{0} \operatorname{Re}(\beta)\right] a^{\dagger} a-i E\left(a-a^{\dagger}\right), \sigma\right\} \\
& +\kappa D[a] \sigma, \\
L_{\mathrm{int}} \sigma= & -i g_{0}\left(\partial_{\beta} \sigma a^{\dagger} a-\partial_{\beta^{*}} a^{\dagger} a \sigma\right) .
\end{aligned}
$$

The Liouvillian $L_{m}$ affects only the mechanical oscillator and is just the Fokker-Planck version of Eq. (7). A crucial point in this formalism is that the nonlinear optomechanical interaction $L_{\text {int }}$ from Eq. (9) makes a contribution to both the new Liouvillian for the cavity $\mathcal{L}_{c}$ and the new interaction $\mathcal{L}_{\text {int }}$. Parts of the interaction can thus formally be treated as a shift of the detuning by $2 g_{0} \operatorname{Re}(\beta)$ that depends on the phase-space variables $\left(\beta, \beta^{*}\right)$. Note that Eq. (12) is still exactly equivalent to Eq. (6).

A semipolaron transformation.-The parametric dependence of the cavity detuning on the phase-space variables can be transformed into one of the driving field $E$ by means of a transformation

$$
\begin{aligned}
\tilde{\sigma}\left(\beta, \beta^{*}, t\right) & =e^{\eta\left(\beta-\beta^{*}\right) a^{\dagger} a / 2} \sigma\left(\beta, \beta^{*}, t\right) e^{-\eta\left(\beta-\beta^{*}\right) a^{\dagger} a / 2} \\
& =e^{i \theta\left(\beta, \beta^{*}\right) a^{\dagger} a} \sigma\left(\beta, \beta^{*}, t\right) e^{-i \theta\left(\beta, \beta^{*}\right) a^{\dagger} a},
\end{aligned}
$$

with

$$
\theta\left(\beta, \beta^{*}\right)=\eta \operatorname{Im}(\beta), \quad \eta=\frac{2 g_{0}}{\omega_{m}} .
$$

When transforming the equation of motion (12), care has to be taken on commuting the unitary operators in Eq. (15) with derivatives with respect to $\left(\beta, \beta^{*}\right)$ in $L_{m}$ and $L_{\text {int }}$ due to the $\beta$ dependence of $\theta$. Details are given in Appendix C. The resulting equation of motion for $\tilde{\sigma}\left(\beta, \beta^{*}, t\right)$ can be written again in the form of Eq. (12):

$$
\partial_{t} \tilde{\sigma}\left(\beta, \beta^{*}, t\right)=\left(L_{m}+\tilde{\mathcal{L}}_{c}+L_{\mathrm{int}}\right) \tilde{\sigma}\left(\beta, \beta^{*}, t\right),
$$

where $L_{m}$ and $L_{\text {int }}$ remain unchanged as in Eqs. (13) and (14), and the Liouvillian operator for the cavity becomes

$$
\begin{aligned}
\tilde{\mathcal{L}}_{c} \tilde{\sigma}= & -i\left[-\Delta a^{\dagger} a-K\left(a^{\dagger} a\right)^{2}-i E\left(e^{-i \theta\left(\beta, \beta^{*}\right)} a-\text { H.c. }\right), \tilde{\sigma}\right] \\
& +\kappa D[a] \tilde{\sigma} .
\end{aligned}
$$

In this picture, the phase of the driving field is different for each point in phase space [via $\theta\left(\beta, \beta^{*}\right)$ ], and the cavity acquires an effective Kerr nonlinearity of strength

$$
K=\frac{g_{0}^{2}}{\omega_{m}} .
$$

We point out that the effective Kerr nonlinearity of the optomechanical interaction gives rise to ponderomotive squeezing of light, as was recently observed in Refs. [6,7].

The equation of motion for $\tilde{\sigma}\left(\beta, \beta^{*}, t\right)$ [Eq. (16)] is an approximation. In principle, it contains further terms that are of order $Q_{m}^{-1}$ and whose explicit form is given in Appendix A1. For high-quality oscillators, these terms provide only small corrections and, therefore, will be dropped in the following. Apart from this approximation, Eq. (16) still contains the full nonlinear dynamics of the system, while the aspect of the optical Kerr nonlinearity is explicitly separated from the nonlinearity in the 
optomechanical interaction. It is also important to note that the quasiprobability distribution for the reduced state of the oscillator still follows from the transformed state $\tilde{\sigma}\left(\beta, \beta^{*}, t\right)$ in Eq. (15) by taking the partial trace over the cavity

$$
Q\left(\beta, \beta^{*}\right)=\operatorname{tr}_{c}\left\{\tilde{\sigma}\left(\beta, \beta^{*}\right)\right\}
$$

Semipolaron versus polaron transformation.-The transformation in Eq. (15) has many parallels with the polaron transformation [39] that has been applied fruitfully to optomechanical systems in order to describe singlephoton strong-coupling effects $[34,35]$. The polaron transformation is effected by a unitary transformation of the density operator

$$
\tilde{\rho}_{\mathrm{pol}}=e^{\eta\left(b-b^{\dagger}\right) a^{\dagger} a / 2} \rho e^{-\eta\left(b-b^{\dagger}\right) a^{\dagger} a / 2}
$$

that should be compared to the transformation in Eq. (15). Instead of fulfilling Eq. (6), the transformed state $\tilde{\rho}$ fulfills a transformed master equation

$$
\begin{gathered}
\dot{\tilde{\rho}}_{\mathrm{pol}}=\left(L_{m}+\tilde{L}_{c}\right) \tilde{\rho}_{\mathrm{pol}}, \\
\tilde{L}_{c} \tilde{\rho}_{\mathrm{pol}}=-i\left[-\Delta a^{\dagger} a-K\left(a^{\dagger} a\right)^{2}\right. \\
\left.-i E\left(e^{-\eta\left(b-b^{\dagger}\right) / 2} a-\text { H.c. }\right), \tilde{\rho}_{\mathrm{pol}}\right] \\
+\kappa D\left[e^{-\eta\left(b-b^{\dagger}\right) / 2} a\right] \tilde{\rho}_{\mathrm{pol}},
\end{gathered}
$$

where $L_{m}$ is given in Eq. (7). This equation is again correct up to terms of order $Q_{m}^{-1}$. It is instructive to compare the master equation in the polaron picture (20) to the equation of motion (16) attained in our "semipolaron transformation." In both equations of motion, the Liouvillians for the cavity [Eqs. (17) and (21), respectively] exhibit a Kerr nonlinearity and contain a driving field whose phase depends on the momentum of the oscillator. Crucially, the polaron transformation changes the jump operator describing cavity decay from $a$ to $e^{i \eta\left(b-b^{\dagger}\right) / 2} a$ and entirely removes the interaction term (9). Moreover, since the polaron picture corresponds to a transformation into dressed states of the optomechanical system, the partial trace of $\tilde{\rho}_{\text {pol }}$ over the (dressed) cavity mode does not give the reduced state of the mechanical oscillator; cf. Eq. (19). In contrast, the semipolaron transformation introduced here retains a nonlinear interaction $L_{\text {int }}$ [Eq. (14)], leaves the jump operator for cavity decay unchanged, and conserves the important relation (18). These properties are crucial in order to perform second-order perturbation theory in $L_{\text {int }}$ and to derive an effective equation of motion for the mechanical oscillator as a separate system. For further comments on the semipolaron transformation, we refer to Appendix C.

\section{B. Fokker-Planck equation for the mechanical oscillator}

Interaction picture.-Our goal is now to adiabatically eliminate the cavity field from the dynamics, similar to the analysis of sideband cooling [36]. This elimination requires that the cavity dynamics, governed by $\tilde{\mathcal{L}}_{c}$ in Eq. (17) with a dominant characteristic time scale $\kappa$, is fast as compared to all other time scales in $\mathcal{L}_{m}$ and $\mathcal{L}_{\text {int }}$. Since we aim to also cover, in particular, the resolved-sideband regime $\omega_{m}>\kappa$, we move to an interaction picture with respect to the free harmonic motion of the mirror. The equation of motion is still given by Eq. (16), where $L_{m}$ describes thermal decay only:

$$
L_{m} \sigma=\gamma\left[\partial_{\beta} \beta+\partial_{\beta^{*}} \beta^{*}+2(\bar{n}+1) \partial_{\beta \beta^{*}}^{2}\right] \sigma,
$$

and $\tilde{\mathcal{L}}_{c}$ and $L_{\text {int }}$ become explicitly time dependent:

$$
\begin{aligned}
\tilde{\mathcal{L}}_{c} \sigma= & -i\left[-\Delta a^{\dagger} a-K\left(a^{\dagger} a\right)^{2}-i E\left(e^{-i \theta\left(\beta, \beta^{*}, t\right)} a-\text { H.c. }\right), \sigma\right] \\
& +\kappa D[a] \sigma, \\
L_{\text {int }} \sigma= & -i g_{0}\left(e^{i \omega_{m} t} \partial_{\beta} \sigma a^{\dagger} a-\text { H.c. }\right) .
\end{aligned}
$$

The phase of the driving field is $\theta\left(\beta, \beta^{*}, t\right)=\eta \operatorname{Im}\left(\beta e^{-i \omega_{m} t}\right)$.

In the adiabatic elimination, it is assumed that the cavity essentially remains in the (quasi)stationary state of its undisturbed (by $L_{\text {int }}$ ) dynamics

$$
\dot{\rho}_{c}=\tilde{\mathcal{L}}_{c} \rho_{c},
$$

with $\tilde{\mathcal{L}}_{c}$ given by Eq. (22). This Liouvillian describes the dynamics of a Kerr nonlinear cavity driven by an amplitude- and phase-modulated field

$$
E e^{i \theta\left(\beta, \beta^{*}, t\right)}=E \sum_{n=-\infty}^{\infty} J_{n}(-\eta|\beta|) e^{\operatorname{in}\left(\omega_{m} t-\varphi\right)},
$$

where $J_{n}$ are Bessel functions. Note that the partial amplitudes depend on the mechanical phase-space variable $\beta=|\beta| e^{i \phi}$. We do not attempt to exactly solve Eq. (23). While, in fact, an exact solution for the stationary state of a Kerr nonlinear cavity exists [40] for the case of a constant driving field (i.e., $\theta \equiv$ const), no such state can be expected for the present situation. Because of the periodic modulation of the driving field, the cavity will not settle into a strictly stationary state but rather to a quasistationary state with a periodic time dependence. If the Kerr nonlinearity is neglected, an exact solution for this quasistationary state can be constructed by means of a Floquet-series ansatz [41]. However, in the present case, both aspects, modulated drive and Kerr nonlinearity, are important and shall be taken into account.

In order to arrive at an approximate solution of Eq. (23) that can serve as a ( $\beta$-dependent) reference state for the adiabatic elimination of the cavity, we will follow two 
complementary approaches in the paragraphs below: In the first one, we assume the cavity is driven to a state of large mean amplitude that we determine self-consistently from an essentially classical nonlinear dynamics. The fluctuations around this mean field will be treated in a linearized model as Gaussian noise. The second approach concerns the case of weak driving fields for which the cavity essentially stays close to its ground (vacuum) state that corresponds to the regime considered in Refs. [25,26]. In this case, the master equation [Eq. (23)] can be expanded and directly solved on the low-lying Fock states.

In both cases, we aim to retain a nonlinear dynamics for the mean cavity amplitude and use a linearized description for fluctuations. Formally, this derivation is done by switching to a displaced frame, defining $\tilde{\tilde{\sigma}}=D[\alpha(t)] \tilde{\sigma} D^{\dagger}[\alpha(t)]$, where $D(\alpha)=\exp \left(\alpha a^{\dagger}-\alpha a\right)$. We choose $\alpha(t) \in \mathbb{C}$ such that the terms of dominant order in $\alpha$ are canceled from the transformed master equation for $\tilde{\tilde{\sigma}}$. In the case of $|\alpha(t)| \gg 1[|\alpha(t)| \ll 1]$, we cancel the terms of third [up to first] order in $\alpha$ and then neglect the terms up to first [of third] order in $\alpha$. The full equation of this transformation may be found in Eq. (A13) of Appendix A; we proceed here with its most important features.

Displaced frame for the limit $|\alpha(t)| \gg 1$.- In the limit $|\alpha(t)| \gg 1$, we identify $\alpha(t)$ with the long-time solution of

$$
\dot{\alpha}(t)=-\left\{\kappa-i\left[\Delta+2 K|\alpha(t)|^{2}\right]\right\} \alpha(t)+E e^{i \theta\left(\beta, \beta^{*}, t\right)}
$$

that formally follows from the requirement that terms of third order in $\alpha$ in the resulting master equation for the displaced state $\tilde{\tilde{\sigma}}$ are canceled. Because of the Kerr nonlinearity, the dynamics described by this equation of motion can be bistable. On assuming a single stable solution, we preclude bistable regimes from our description. For a constant phase $\theta$, bistability occurs only for driving fields that are red detuned with respect to the cavity resonance for detunings $\Delta<-\sqrt{3} \kappa$; see Ref. [40]. For the present case of a modulated drive, no such simple condition can be given. However, it is reasonable to expect that bistability will become an issue only when the driving field has sufficient spectral weight for frequencies with a detuning below $-\sqrt{3} \kappa$. In the following, we are mainly concerned with the cases of resonant or blue detuned drive, for which it turns out that bistability is not an issue [42-44].

From Eqs. (25) and (24), we can expect that in the longtime limit, the cavity amplitude will be of the form

$$
\alpha\left(\beta, \beta^{*}, t\right)=\sum_{n=-\infty}^{\infty} \alpha_{n}\left(\beta, \beta^{*}\right) e^{\mathrm{in} \omega_{m} t}
$$

Inserting this expression into Eq. (25), one sees that the effective detuning experienced by the cavity will be dominantly given by the dc component of $|\alpha(t)|^{2}$, such that it is useful to define an effective detuning

$$
\Delta_{\text {eff }}\left(\beta, \beta^{*}\right)=\Delta+2 K \sum_{n}\left|\alpha_{n}\left(\beta, \beta^{*}\right)\right|^{2} .
$$

Equation (27) has to be read as a nonlinear equation for $\Delta_{\text {eff }}$. In regimes where more than one solution exists, the system will be bi-or multistable, and we have to expect large photon-number fluctuations. The validity of our approach will thus be limited to regions where only a single stable solution for $\Delta_{\text {eff }}$ exists, as discussed above. We seek an approximate solution to Eq. (25) by assuming a fixed effective detuning $\Delta_{\text {eff }}$, such that

$$
\begin{aligned}
& \alpha_{n}=\frac{E}{h_{n}} J_{n}(-\eta|\beta|) e^{-i n \phi}, \\
& h_{n}=\kappa+i\left(n \omega_{m}-\Delta_{\text {eff }}\right),
\end{aligned}
$$

where we follow the notation of Refs. [25,26]. In total, $\alpha(t)$ in Eq. (26) depends on the mechanical phase-space variable $\beta$ through both $\Delta_{\text {eff }}\left(\beta, \beta^{*}\right)$ and the $\beta$-dependent driving field $E e^{i \theta\left(\beta, \beta^{*}, t\right)}$. We will see that the $\beta$ dependence in $\Delta_{\text {eff }}\left(\beta, \beta^{*}\right)$ is a crucial effect for the case of resonant cavity drive (for which $\Delta_{\text {eff }} \lesssim \kappa$ ).

The Liouvillians after the transformation with $D[\alpha(t)]$ are

$$
\begin{aligned}
L_{m} \sigma= & \gamma\left[\partial_{\beta} \beta+\partial_{\beta^{*}} \beta^{*}+2(\bar{n}+1) \partial_{\beta \beta^{*}}^{2}\right] \sigma \\
& -i g_{0}\left[\partial_{\beta} e^{i \omega_{m} t}|\alpha(t)|^{2}-\text { H.c. }\right] \sigma
\end{aligned}
$$

$$
\begin{aligned}
\tilde{\mathcal{L}}_{c} \sigma= & -i\left\{-\left[\Delta+4 K|\alpha(t)|^{2}\right] a^{\dagger} a-K\left[\alpha(t)^{2} a^{\dagger 2}+\text { H.c. }\right], \sigma\right\} \\
& +\kappa D[a] \sigma \\
L_{\mathrm{int}} \sigma & =-i g_{0}\left\{e^{i \omega_{m} t} \partial_{\beta} \sigma\left[\alpha^{*}(t) a+\alpha(t) a^{\dagger}\right]+\text { H.c. }\right\} .
\end{aligned}
$$

The Liouvillian for the mechanical oscillator $L_{m}$ acquires an additional drift term [second line in Eq. (30)] with a nonlinear drift coefficient $\alpha e^{i \omega_{m} t}\left|\alpha\left(\beta, \beta^{*}, t\right)\right|^{2}$ that contains, in particular, the nonlinear dc force and dynamic backaction effects (i.e., optical damping and frequency shifts), $\underset{\sim}{\text { as }}$ will be discussed below. In the Liouvillian for the cavity $\tilde{\mathcal{L}}_{c}$, terms of order $\alpha(t)$ and lower are dropped. The leading terms of order $\alpha^{2}$ describe squeezing dynamics and an effective detuning. Finally, in $L_{\text {int }}$, only the term of linear order in $\alpha$ is kept. Note also that when moving to the displaced frame, commutators of the ( $\beta$-dependent) displacement operators and derivatives with respect to $\beta$ have been neglected. They would add corrections to the Liouvillians of higher order in $g_{0}$. We have now removed the driving field from the dynamics of the cavity. The remaining Liouvillian (31) describes the Gaussian evolution of fluctuations.

The ponderomotive squeezing of the light field is naturally contained in the $\alpha^{2} a^{\dagger 2}$ term and its Hermitian conjugate. While in this article we will study parameters for 
which this squeezing is negligible, the effect of ponderomotive squeezing back on the mirror after the adiabatic elimination of the cavity is an interesting perspective for future applications of our new formalism: In the adiabatic elimination of the cavity, one would have to use a squeezed reference state that can possibly introduce additional diffusion terms in the motion of the mirror. Applied to the situation of limit cycles, this additional diffusion may cause the state of the oscillator to become more classical.

Curiously, the Kerr nonlinearity induces a different effective detuning for the mean field $\alpha$ than for the fluctuations [compare Eqs. (25) and (31)]. This is consistent with results for a Kerr nonlinear cavity [40]. We therefore define

$$
\tilde{\Delta}_{\text {eff }}=\Delta+4 K \sum_{n}\left|\alpha_{n}\right|^{2}
$$

The fast decay rate to the vacuum is still given by $\kappa$ from the original master Eq. (8).

This fact can be used in order to adiabatically eliminate the cavity, taking into account second-order effects in the optomechanical interaction $\propto g_{0}$ [Eq. (32)], very much in the spirit of laser-cooling theory [36]. Details of the calculation can be found in Appendix B. The result is an effective equation of motion for the mechanical oscillator in the form of a Fokker-Planck equation

$$
\begin{aligned}
\dot{Q}\left(\beta, \beta^{*}\right)= & g_{0}^{2} \sum_{n}\left(\partial_{\beta^{*}} \partial_{\beta} \frac{\alpha_{n}^{*} \alpha_{n}}{\tilde{h}_{n-1}}-\partial_{\beta^{*}} \partial_{\beta^{*}} \frac{\alpha_{n-2}^{*} \alpha_{n}}{\tilde{h}_{n-1}}\right) Q\left(\beta, \beta^{*}\right) \\
& + \text { H.c. }+i g_{0} \sum_{n}\left(\partial_{\beta^{*}} \alpha_{n-1}^{*} \alpha_{n}\right) Q\left(\beta, \beta^{*}\right)+\text { H.c. } \\
& +\gamma\left[\partial_{\beta} \beta+\partial_{\beta^{*}} \beta^{*}+2(\bar{n}+1) \partial_{\beta \beta^{*}}^{2}\right] Q\left(\beta, \beta^{*}\right)
\end{aligned}
$$

for the $Q$ function of the mechanical oscillator. In analogy to $h_{n}$, we define $\tilde{h}_{n}=\kappa+i\left(n \omega_{m}-\tilde{\Delta}_{\text {eff }}\right)$, with $\tilde{\Delta}_{\text {eff }}$ given in Eq. (33).

The drift and diffusion coefficients in the Fokker-Planck equation (34) do not depend on the phase of $\beta$ as a consequence of the rotating-wave approximation involved in its derivation. We therefore transform the Fokker-Planck equation to polar coordinates $\beta=r e^{i \phi}$ and focus on the time evolution of the oscillator amplitude $r$ by integrating out the phase variable $\phi$. The time evolution for $r$ is then a one-dimensional Fokker-Planck equation (on a half-space)

$$
\dot{Q}(r)=-\partial_{r} \mu(r) Q(r)+\partial_{r}^{2} D(r) Q(r)
$$

with drift $\mu(r)$ and diffusion coefficient $D(r)$

$$
\mu(r)=-\gamma r+\sum_{n} g_{0} E^{2} \operatorname{Im}\left[\frac{J_{n-1}(\eta r) J_{n}(\eta r)}{h_{n-1} h_{n}^{*}}\right],
$$

$$
\begin{aligned}
D(r)= & \frac{\gamma(\bar{n}+1)}{2}+\sum_{n} \frac{g_{0}^{2} E^{2}}{2} \\
& \times\left(\frac{\kappa J_{n}(\eta r)^{2}}{\left|h_{n}\right|^{2}\left|\tilde{h}_{n-1}\right|^{2}}-\operatorname{Re}\left[\frac{J_{n-2}(\eta r) J_{n}(\eta r)}{\tilde{h}_{n-1} h_{n-2}^{*} h_{n}}\right]\right) .
\end{aligned}
$$

The details of the transformation may again be found in Appendix B. Equation (35) admits a potential solution in the steady state that is given by (up to normalization)

$$
Q(r) \propto \frac{e^{I(r)}}{D(r)}, \quad I(r):=\int_{0}^{r} \frac{\mu\left(r^{\prime}\right)}{D\left(r^{\prime}\right)} d r^{\prime} .
$$

This solution is valid for any value of $\Delta$, such that it covers both the regime of optomechanical cooling and the regime of self-induced oscillations. In Ref. [36], an effective equation of motion for the oscillator was derived in order to study the limits of sideband cooling under linearization of the dynamics and adiabatic elimination of the cavity using a coherent state as a reference state. The present approach generalizes this calculation to the nonlinear regime by using a different reference state for each phase-space point of the oscillator. The nonlinear quantum dynamics has been described analytically using a method based on the classical theory for limit cycles [45] and by means of Langevin equations [25], and has been applied in great detail to limit cycles but also to the cooling regime [26]. The results of our calculation reproduce these results in the regime of a negligible Kerr term and provide suitable extensions in those cases where the Kerr nonlinearity of the cavity becomes a dominant effect. In the next section, we will compare the analytical expression for the steady state of the mechanical to numerical solutions of the exact master equation (6) to study the limit-cycle regime. We conclude this section by briefly stating the corresponding results for the limit of small intracavity field amplitude, followed by a comparison of limit cycles studied in different laser setups.

Displaced frame and adiabatic elimination for the limit $|\alpha(t)| \ll 1$. - In the case of $|\alpha(t)| \ll 1$, all steps can be performed in analogy. The difference is that we need to cancel the terms up to first order in $\alpha$ and then neglect the terms of third order in $\alpha$. The effective detuning is now given by

$$
\Delta_{\text {eff }}=\Delta+K,
$$

i.e., the bare detuning is just shifted by the constant Kerr term in this extreme regime. No distinction between $\Delta_{\text {eff }}$ and $\tilde{\Delta}_{\text {eff }}$ needs to be made. The adequate choice for the displacement amplitude is the long-term solution of

$$
\dot{\alpha}(t)=-\left[\kappa-i \Delta_{\mathrm{eff}}\right] \alpha(t)+E e^{i \theta\left(\beta, \beta^{*}, t\right)} .
$$


The result of the adiabatic elimination is structurally the same; $\alpha_{n}$ and $h_{n}$ are given as in Eqs. (28) and (29) but with the effective detuning now given as in Eq. (39). In Eq. (37), the $\tilde{h}_{n}$ are simply replaced by $h_{n}$.

Quantum limit cycles in lasers.-It seems natural to base a model of optomechanical limit cycles on theory used in the context of lasers [30-32], where quantum limit cycles have been extensively studied most prominently. The standard laser system consists of a reservoir of many atoms that forms a bath for the cavity mode. The pumped atoms will drive the laser mode to a high-amplitude limit cycle, where it settles into a coherent state with random phase.

A setup that can be driven to highly sub-Poissonian states is the regularly pumped laser [46,47], where excited atoms fly through a cavity. The mechanism works in the situation, where at each time approximately only one atom interacts with the light mode and the interaction is a swapping of excitations. In the case of more regular than Poissonian statistics of the pump, the fluctuation of the transmitted energy decreases and the light mode will have sub-Poissonian phonon statistics. This setup is sometimes also referred to as a one-atom laser, or as a micromaser, because the events when more than one atom interacts with the field can be neglected.

The one-atom laser is different from the one-and-thesame-atom laser [48], where a single atom is trapped inside a cavity and drives the laser mode. Also, in this setup, a sub-Poissonian steady state can be reached, and the explanation again relies on counting the number of interactions exchanged between the atom and the cavity [49].

In our optomechanical system, a single laser mode is the bath driving the mechanical oscillator. The bath consisting of only a single mode is in analogy to some extent to the micromaser, as stressed in Ref. [28], and is even more similar to the one-and-the-same-atom laser. Even though we also describe sub-Poissonian boson statistics, the analytical techniques developed, e.g., in Ref. [49] cannot be readily applied to our situation because they crucially rely on the preservation of total excitations by the interaction, which is not given in the optomechanical setup. Our analytical model in Sec. III is based on Ref. [31], which was first developed for the standard setup without subPoissonian statistics.

For the creation of non-Gaussian states, a nonlinearity is required. In the optomechanical setup, the nonlinearity stems from the interaction, while in the one-and-the-sameatom laser, it stems from the two-level nature of the bath, which is equivalent to a highly nonlinear cavity.

\section{OPTOMECHANICAL LIMIT CYCLES IN THE QUANTUM REGIME}

\section{A. Introduction}

As an introduction to our study, we sum up some known results on limit cycles that the rest of the article refers to.
First, we introduce the theory for the amplitude of classical limit cycles, as developed in Refs. $[19,23]$, and then we recapitulate the numerical results on nonclassical states of quantum limit cycles, as reported in Ref. [27]. When comparing these findings to our analytical treatment, we will mainly be concerned with the special case of the close to resonant driving field $\Delta_{\text {eff }} \simeq \kappa \ll \omega_{m}$. Therefore, we start out by stating some approximate expressions for this case.

Close to resonant drive.-In the sideband-resolved regime and with a detuning close to the resonance, i.e., $\Delta_{\text {eff }}, \kappa \ll \omega_{m}$ (but not necessarily $\Delta_{\text {eff }} \ll \kappa$ ), we keep only the terms with $n=0,1$ in the expression for the drift coefficient [Eq. (36)] and approximate

$$
\mu(r) \simeq-\gamma r+\frac{g_{0} E^{2}}{\omega_{m}^{2}} \frac{2 \kappa \Delta_{\mathrm{eff}}(r)}{\Delta_{\mathrm{eff}}^{2}(r)+\kappa^{2}} J_{0}(\eta r) J_{1}(\eta r) .
$$

In the sideband-resolved regime, the equation for the effective detuning [Eq. (27)] becomes a third-order polynomial in $\Delta_{\text {eff }}$, and in the limit $\Delta_{\text {eff }} \ll \kappa$, it even simplifies to a simple and explicit expression

$$
\Delta_{\text {eff }}(r) \simeq \Delta+2 \frac{K E^{2}}{\kappa^{2}} J_{0}^{2}(\eta r) .
$$

Classical limit cycles.-The theory for classical optomechanical limit cycles from Ref. [19] is reproduced by the drift part of the Fokker-Planck equation [Eq. (36)] when neglecting the diffusion and using a constant effective detuning $\Delta_{\text {eff }}(r)=\Delta_{\text {eff }} \equiv$ const. Disregarding the diffusion, the oscillator amplitude $r(t)$ evolves fully deterministically and obeys

$$
\dot{r}=\mu(r)=-\gamma_{\mathrm{eff}}(r) r, \quad \gamma_{\mathrm{eff}}(r)=\gamma+\gamma_{\mathrm{opt}}(r) .
$$

Following Eq. (41), the combined intrinsic and optically induced damping of the oscillator $\gamma_{\mathrm{eff}}(r)$ close to resonance is then given as the sum of the intrinsic mechanical damping $\gamma$ and the amplitude-dependent optical damping

$$
\gamma_{\mathrm{opt}}(r)=-\frac{g_{0} E^{2}}{\omega_{m}^{2}} \frac{2 \kappa \Delta_{\mathrm{eff}}}{\Delta_{\mathrm{eff}}^{2}+\kappa^{2}} \frac{J_{0}(\eta r) J_{1}(\eta r)}{r} .
$$

Note that the sign of the optically induced damping at $r=0$ coincides with the sign of $\Delta_{\text {eff }}$. For negligible intrinsic damping $\gamma \ll \gamma_{\mathrm{opt}}$, one can then expect limit cycles to always start for $\Delta_{\text {eff }}>0$ (whereas the dynamics will be stable for $\Delta_{\text {eff }}<0$ ). The possible amplitudes $r_{0}$ for limit cycles are given by the conditions $\gamma_{\text {eff }}\left(r_{0}\right)=0$ and $\gamma_{\text {eff }}^{\prime}\left(r_{0}\right)>0$. The first condition is equivalent to

$$
\frac{J_{0}(\eta r) J_{1}(\eta r)}{r}=\gamma \frac{\omega_{m}^{2}}{g_{0} E^{2}} \frac{\Delta_{\text {eff }}^{2}+\kappa^{2}}{2 \kappa \Delta_{\text {eff }}} .
$$



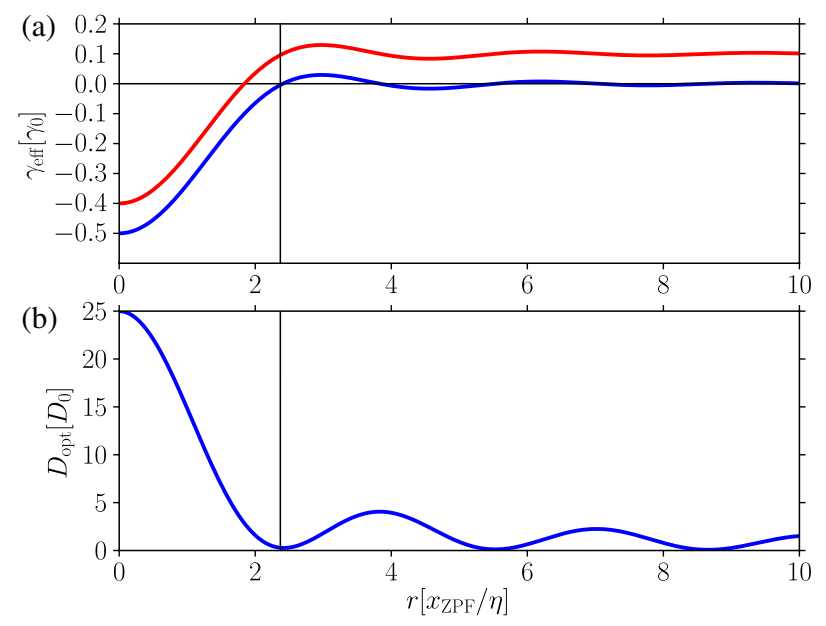

FIG. 1 (color online). (a) Effective damping $\gamma_{\text {eff }}(r)=\gamma+\gamma_{\text {opt }}(r)$ from Eq. (43) in units of $\gamma_{0}=\left(g_{0} E^{2} / \omega_{m}^{2}\right) /\left(2 \kappa \Delta_{\text {eff }} / \Delta_{\text {eff }}^{2}+\kappa^{2}\right)$ versus cycle amplitude $r$ in units of zero-point fluctuation and $\eta$. The blue and red lines are two examples for different intrinsic dampings $\gamma$. Limit cycles are stable at roots of the total damping with a positive slope. This condition is fulfilled only once for the red line with $\gamma=0.1 \gamma_{0}$, corresponding to only one possible amplitude for the oscillation. For the blue line with $\gamma=0$, many such intersections occur and the oscillator amplitude will, in general, jump between those different metastable points. (b) The optical part of the diffusion from approximation (48) for $\kappa / \omega_{m}=0.1$ and $\Delta_{\text {eff }}=\kappa$ in units of $D_{0}=\kappa g_{0}^{2} E^{2} / \omega_{m}^{4}$. Note that the dominant part of the diffusion from Eq. (48) is exactly canceled at the position of the limit cycle for $\gamma=0$, as indicated by the vertical line. This cancellation explains the strongly sub-Poissonian phonon statistics for such parameters.

The left-hand side of this equation has infinitely many roots, as the Bessel functions oscillate at a constant amplitude; cf. Fig. 1(a). The envelope is given by the $r^{-1}$ decay. As illustrated in Fig. 1(a), the exact position of the limit cycle and the number of possible amplitudes are then determined by the right-hand side of Eq. (44).

\section{B. Outline}

In the following subsections, we will explain two features of limit cycles on resonance that can be heavily influenced by the Kerr term.

First, in Sec. IVC, we show that in the strong driving limit $|\alpha|^{2} \gg 1$, the phase transition between optomechanical cooling and self-induced oscillations is crucially determined by the dynamical dependence of the effective detuning on the intracavity amplitude and its corresponding nonlinear dependence on the cycle amplitude; cf. Eq. (42). This behavior can also be explained in a classical picture.

We then develop an explanation of the interesting numerical result for limit cycles in the quantum regime reported in Refs. [27,28]: For approximately resonant driving fields $\Delta \simeq 0$, and at the blue detuned sideband resonance $\Delta \simeq \omega_{m}$, the steady state of the mechanical

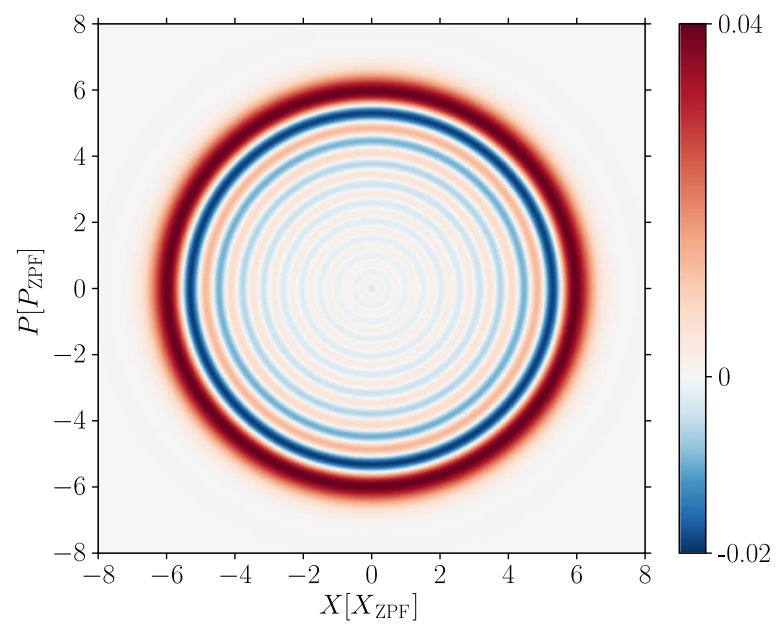

FIG. 2 (color online). Wigner function $W$ of the lowest metastable limit cycle of the mechanical oscillator for parameters $\left(g_{0}, \kappa_{E}=\right.$ $2 \kappa, \gamma, E, \Delta, K)=(0.275,0.1,0,0.15,-0.026,0.076) \times \omega_{m} . \quad$ As there are fewer than 0.03 photons in the cavity, we are in the regime of $|\alpha|^{2} \ll 1$, where $\Delta_{\text {eff }}=\Delta+K$; cf. Eq. (39). Choosing the bare detuning to minimize the Fano factor $(F=0.1$ at the attractor with lowest amplitude, which is depicted in this plot) implies that according to Eq. (50), $\Delta=\kappa-K$, which for the strong optomechanical coupling of this example gives the negative numerical value $\Delta=-0.026$. Note that classically or excluding the Kerr effect, a limit cycle would not even start for these parameters. The minimal value of $W$ in this plot is -0.02 .

oscillator can have a Wigner function with a negative area. The requirement on the strength of the optomechanical coupling $g_{0}$ is more stringent at the sideband than on resonance, where nonclassical limit cycles already appear for weaker coupling. Curiously, on resonance, the numerical solution to the master equation also predicts (nonclassical) limit cycles for parameters where classically the effective detuning $\Delta_{\text {eff }}<0$, and one would expect a stable cooling dynamics. Figure 2 shows the steady-state Wigner function of the mechanical oscillator for such parameters.

We will use the analytical description of limit cycles with the Fokker-Planck equation to explain the features displayed in Fig. 2 and to predict general requirements on system parameters to achieve a nonpositive Wigner function. In Sec. IVE, we show that the occurrence of negative Wigner functions, in turn, is intimately linked to achieving a small variance of the phonon statistics, as characterized by a small Fano factor $F=\langle\Delta n\rangle^{2} /\langle n\rangle$, along with a small cycle amplitude $r_{0}$. We analyze the variance of the phonon number in Sec. IVD and find that the conditions for a small Fano factor are favorable at the $\Delta=0$ resonance.

In Sec. IVF, we describe the numerical method used to check the analytical predictions. It allows us for the first time to numerically study quantum features of optomechanical limit cycles in the regime of large mechanical amplitudes and strong laser drive, populating many states 
of the cavity. We find that the analytical model can still be applied, and even for $g_{0}<\kappa$, the negativity of the Wigner function can be observed.

\section{Drift and dynamical detuning}

In this section, we study in detail the time evolution of the mean amplitude $\bar{r}$, which is determined by the drift $\mu(r)$ in Eq. (41). In particular, we show how the dynamical dependence of $\Delta_{\text {eff }}(r)$ on $r$ gives new results that are not observed in any model based on a static detuning (like the one we used above). We focus on the regime where $\bar{r}$ is larger than its standard deviation $\Delta r$, such that we can derive the time evolution of $\bar{r}$ via $\dot{\bar{r}}=\mu(\bar{r})$ directly from Eq. (41) as

$$
\dot{\bar{r}}=-\gamma \bar{r}+\frac{2 \kappa g E^{2}}{\omega_{m}^{2}} \frac{\Delta_{\mathrm{eff}}(\bar{r})}{\Delta_{\mathrm{eff}}(\bar{r})^{2}+\kappa^{2}} J_{0}(\eta \bar{r}) J_{1}(\eta \bar{r}) .
$$

These assumptions are fulfilled for small $\eta=\frac{2 g_{0}}{\omega_{m}}$ because $\eta \bar{r}$ is the argument of the Bessel functions, and hence $\bar{r} \propto \frac{1}{\eta}$.

With the oscillator initially in the ground state, it is the sign of $\Delta_{\text {eff }}(0)$ that determines if the limit cycle starts at all: For $\Delta_{\text {eff }}(0)<0$, the optical damping is initially positive and no oscillation starts, but for $\Delta_{\text {eff }}(0)>0$, it is negative and may be larger than the intrinsic damping $\gamma$, so that selfinduced oscillations can start. The oscillator arrives at its steady state, when $\dot{\bar{r}}=0$. Neglecting the small corrections due to $\gamma$, this is equivalent to the condition $\Delta_{\text {eff }}(\bar{r}) J_{0}(\eta \bar{r}) J_{1}(\eta \bar{r})=0$. If the effective detuning $\Delta_{\text {eff }}$ is independent of $r$, the smallest root of this product is always the first root of $J_{0}$. This corresponds to the standard situation (as discussed above) valid for a negligible Kerr parameter or in the weak driving limit; cf. Eq. (39).

In the converse case, for large amplitudes $|\alpha|^{2} \gg 1$ and a non-negligible Kerr parameter, the dynamic nature of the effective detuning can become important: The smallest root of the product $\Delta_{\text {eff }}(\bar{r}) J_{0}(\eta \bar{r}) J_{1}(\eta \bar{r})$ is then determined either by $J_{0}$ or $\Delta_{\text {eff }}$, depending on the sign of $\Delta$. If the bare detuning is on the blue (heating) side $\Delta \gtrsim 0$, the condition for the limit cycle is still $J_{0}\left(\eta r_{0}\right)=0$, as in the case of a static detuning. However, if the bare detuning is on the red (cooling) side $\Delta<0$, the effective detuning for a small cycle amplitude can still be positive as $\Delta_{\text {eff }}(0)=\Delta+2 K E^{2} / \kappa^{2}$; cf. Eq. (42). This situation is the case, in particular, for a driving field $E$ larger than a critical value of $E_{\text {crit }}=\frac{\kappa}{\sqrt{2} g_{0}} \sqrt{|\Delta| \omega_{M}}$. The sign of $\Delta_{\text {eff }}(r)$ will then depend on, and ultimately change with, the increasing amplitude $r$ of the oscillation, since $\Delta_{\text {eff }}=\Delta<0$ at the roots of $J_{0}^{2}(\eta r)$. With increasing oscillator amplitude $r$, the dc component of the cavity occupation and hence (via the Kerr nonlinearity) also the shift of the detuning drop. The steadystate amplitude $r_{0}$ of the limit cycle is reached when $\Delta_{\text {eff }}\left(r_{0}\right)=0$. Using again approximation (42), the condition
$\Delta_{\text {eff }}\left(r_{0}\right)=0$ is equivalent to $J_{0}\left(\eta r_{0}\right)=\frac{\kappa}{\sqrt{2} g_{0} E} \sqrt{|\Delta| \omega_{M}}$. Thus, the Kerr nonlinearity smoothens the transition from cooling to amplification. This smoothing is in contrast to models with a static detuning where a sharp transition occurs at $\Delta_{\text {eff }}=0$.

We numerically check the dynamical nature of the detuning by integrating the equations of motion

$$
\begin{aligned}
& \dot{\alpha}=i \Delta \alpha+g_{0}\left(\beta+\beta^{*}\right) \alpha-\kappa \alpha+E, \\
& \dot{\beta}=i g_{0}|\alpha|^{2}-i \omega_{m} \beta-\gamma \beta
\end{aligned}
$$

that are the classical analogue to the master equation (6). Figure 3 illustrates the time dependence of the detuning with an example of a time evolution where the bare detuning $\Delta<0$, so that the limit-cycle amplitude $r_{0}$ in the steady state is determined by the condition $\Delta_{\text {eff }}(r)=0$. Figure 4 shows that this condition gives a good prediction for $r_{0}$ as a function of $\Delta$.

An approximation similar to Eq. (45) for the case of a laser drive close to the first blue sideband $\Delta \approx \omega_{m}$ shows that there the position of the limit cycle does not depend on the exact value of $\Delta$. It is approximately given by the first root of $J_{1}(\eta r)$. Thus, the limit-cycle amplitude is generally smaller on resonance than on the sideband. We will use this observation in Sec. IVE, where we will see that a small limit-cycle amplitude is favorable for the occurrence of a negative area in the Wigner function.

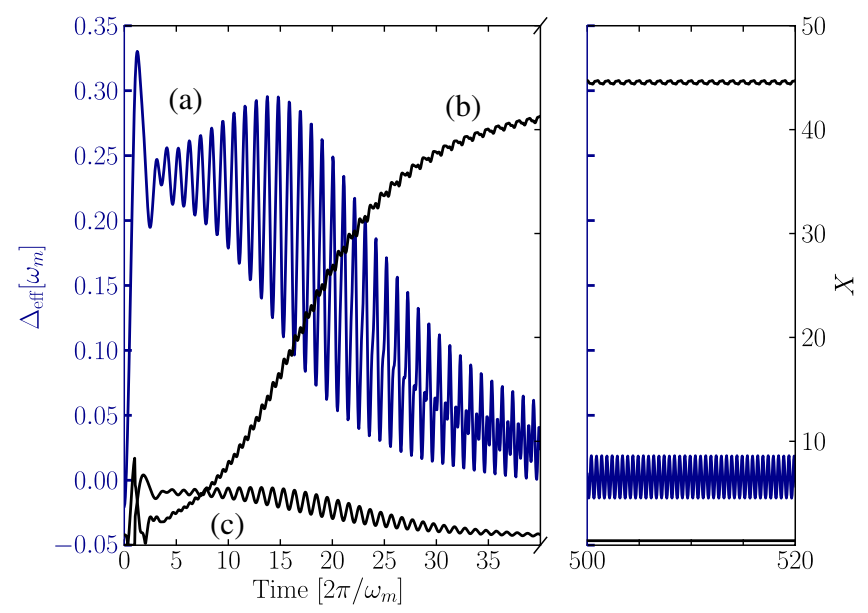

FIG. 3 (color online). Example of the oscillator time evolution for the classical equations of motion [see Eq. (46)], with initial condition $r=0$ for $\Delta \lesssim 0$ but $\Delta_{\text {eff }}(r=0)>0$. (a) Effective detuning $\Delta_{\text {eff }}(t)$ with a scale on the left (blue) axis, (b) oscillator amplitude $r(t)$, and (c) dc shift in position with a scale on the right (black) axis. A positive effective detuning at $r \approx 0$ ensures that the limit cycle starts. With increasing oscillator amplitude, the intracavity photon number $\sum_{n}\left|\alpha_{n}\right|^{2}$ from Eq. (27) drops, and hence also $\Delta_{\text {eff }}$. As $\mu \propto \Delta_{\text {eff }}$ [see Eq. (41)], the oscillator settles into the steady state as soon as this drop reaches $\Delta_{\text {eff }}=0$. The parameters in this plot are $\left(E, g_{0}, \kappa_{E}=2 \kappa, \gamma_{E}=2 \gamma\right)=$ $\left(4.0,0.05,0.3,2 \times 10^{-5}\right) \times \omega_{m}$. 


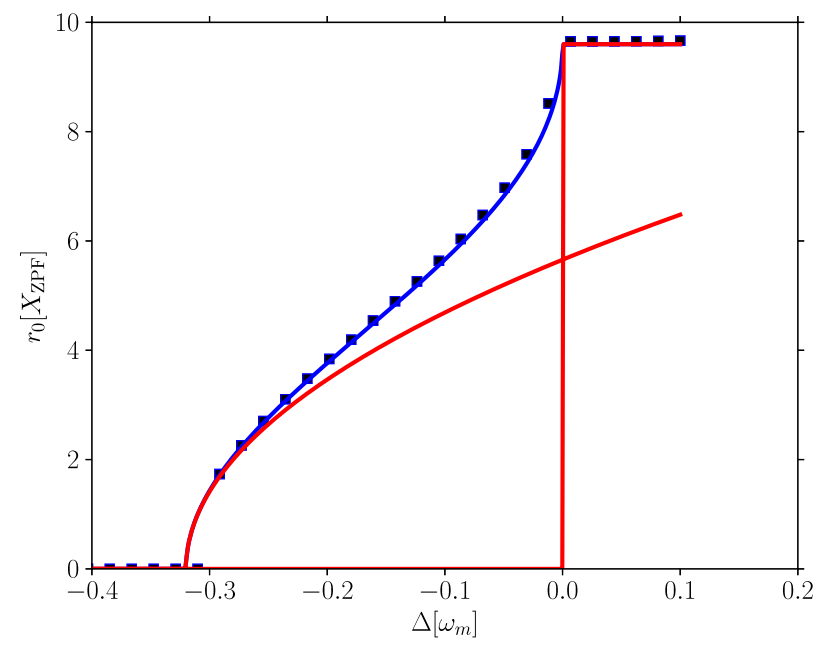

FIG. 4 (color online). Amplitude $r_{0}$ for the first stable limit cycle versus bare detuning $\Delta$. In the limit of an amplitudeindependent effective detuning (red lines), the values for large amplitudes are predicted correctly. It is known from Ref. [19] that for small amplitudes at the onset of limit cycles, the amplitude follows a square root (red lines). With the inclusion of the dynamical effective detuning $\Delta_{\text {eff }}(r)$ (blue line), the limit-cycle amplitude $r_{0}$ follows $J_{0}\left(\eta r_{0}\right)=\frac{\kappa}{\sqrt{2} g_{0} E} \sqrt{|\Delta| \omega_{M}}$, both limit cases are reproduced, and the whole transition between the regimes of damping and antidamping can be described. In this figure, we compare the predictions with the numerical solution (dots) of the classical equation. The parameters of this plot are $\left(E, g_{0}, \kappa_{E}=\right.$ $2 \kappa, \gamma)=(0.5,0.25,0.3,0.0) \times \omega_{m}$.

\section{Diffusion and Fano factor}

Having discussed the conditions for a limit cycle to start and having derived the mean amplitude in the steady state for the $\Delta \simeq 0$ resonance, we now consider the fluctuations caused by the diffusion $D$ around this mean value to derive a prediction for the Fano factor $F=\left(\left\langle n^{2}\right\rangle-\langle n\rangle^{2}\right) /\langle n\rangle=\langle\Delta n\rangle^{2} /\langle n\rangle$, which is a measure for number squeezing: For a coherent state, the phonon distribution is Poissonian, so that $\langle\Delta n\rangle^{2}=\langle n\rangle$ and $F=1$. A state with sub-Poissonian phonon variance can hence be characterized by $F<1$.

We will use the term Fano factor in the context of limit cycles as follows: For generic parameters, an optomechanical system can exhibit several limit cycles, such that the Fano factor of the full density matrix is typically larger than 1. The oscillations at each of these attractors are metastable, such that it is possible to consider the phonon statistics at a particular limit cycle. Especially in the relatively classical regime, where $g_{0} / \omega_{m}$ is not too large, the cycles will be well separated. When we refer to a Fano factor, we will implicitly always mean the Fano factor of one particular attractor.

We obtain the mean and variance of the phonon number $n$ via [32]

$$
\left\langle\left\{a^{r}\left(a^{\dagger}\right)^{s}\right\}_{\mathrm{sym}}\right\rangle=\int d^{2} \alpha W\left(\alpha, \alpha^{*}\right) \alpha^{r}\left(\alpha^{*}\right)^{s},
$$

where $W\left(\alpha, \alpha^{*}\right)$ is the Wigner function. We use here the Wigner function because it gives better agreement with the numerical analysis for the statistics of the phonon number than other quasiprobability distributions. Drift and diffusion coefficients for the Wigner function are calculated in Appendix A along the same lines as shown above for the $Q$ function. In particular, close to resonance, the radial diffusion coefficient as relevant to the Wigner function is

$$
D_{W}=\frac{\gamma(1+2 \bar{n})}{4}+\frac{\kappa g_{0}^{2} E^{2}}{\omega_{m}^{4}}\left(J_{1}^{2}(\eta r)+\frac{1}{2} \frac{\omega_{m}^{2}}{\kappa^{2}+\Delta_{\mathrm{eff}}^{2}} J_{0}^{2}(\eta r)\right)
$$

where we apply to Eq. (B20) the same approximations as in Sec. IVA for the drift coefficient.

For most amplitudes, the $J_{0}^{2}$ term is dominant, as it is enhanced by at least $\left(\omega_{m} / \kappa\right)^{2}$ over the $J_{1}^{2}$ term. For parameters where the optical antidamping is much stronger than the intrinsic mechanical decay, a curious cancellation of the diffusion occurs in the steady state: The limit cycle will then settle exactly at the first root of $J_{0}$, as discussed in Sec. IVC. There, the term proportional to $J_{1}^{2}$, which is suppressed by $\left(\kappa / \omega_{m}\right)^{2}$, becomes the only relevant term in the diffusion. This suppression is illustrated in Fig. 1(b) and can be intuitively explained: The last two terms in Eq. (B21) [or equivalently Eq. (B20)] are the [coherent] squeezing terms. For $n=1$, they exactly cancel the corresponding [incoherent] diffusion terms $\propto \partial_{\beta^{*}} \partial_{\beta^{*}}$ in leading order, and only the higher-order terms in $\kappa^{2} / \omega_{m}^{2}$ remain. Because of this suppression of diffusion in the sideband-resolved regime, one can obtain a very small Fano factor of the mechanical oscillator, as we show below.

The phase-space distribution in the steady state is given by Eq. (38). In the limit of small $g_{0} / \omega_{m}$, where $\Delta n \ll\langle n\rangle$, and for the case of only a single stable limit cycle centered around a position $r_{0}$ with $\mu\left(r_{0}\right)=0$, we linearize $\mu(r) \approx$ $\mu\left(r_{0}\right)+\mu^{\prime}\left(r_{0}\right)\left(r-r_{0}\right)$ around this $r_{0}$ and set $D(r) \simeq D\left(r_{0}\right)$ so that the corresponding solution for $W$ is approximately

$$
W(r) \propto \exp \left(-\frac{\left(r-r_{0}\right)^{2}}{2 \sigma^{2}}\right)
$$

with $\sigma^{2}=-D\left(r_{0}\right) / \mu^{\prime}\left(r_{0}\right)$. One can then derive the approximate expression $F \simeq 4 \sigma^{2}$ for the limit $\omega_{m} / g_{0}>\sigma$. In the sideband-resolved regime and with the limit-cycle position at the first root of $J_{0}$, this gives

$F \simeq\left(\frac{\gamma(1+2 \bar{n})}{4}+\zeta \frac{\kappa g_{0}^{2} E^{2}}{\omega_{m}^{4}}\right) /\left(\frac{\gamma}{4}+\frac{2 \kappa \Delta_{\mathrm{eff}}\left(r_{0}\right)}{\Delta_{\mathrm{eff}}\left(r_{0}\right)^{2}+\kappa^{2}} \zeta \frac{g_{0}^{2} E^{2}}{\omega_{m}^{3}}\right)$, 
(a)

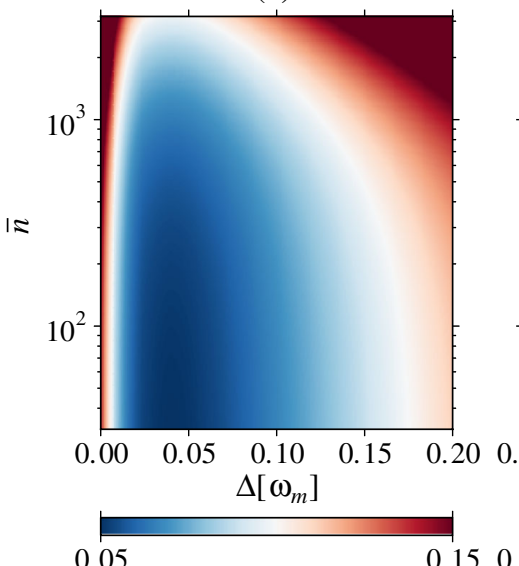

(b)

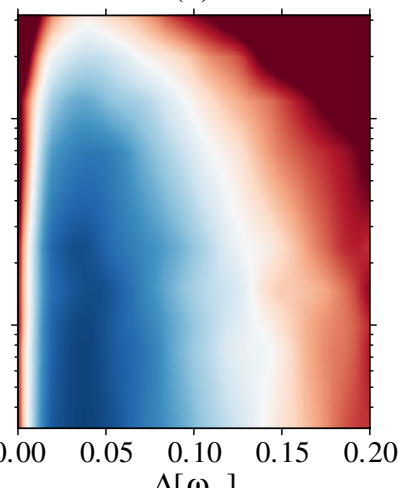

(c)

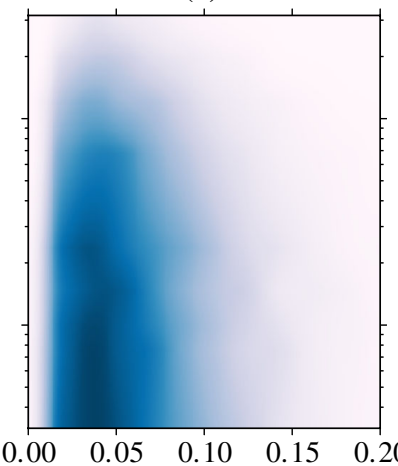

(d)

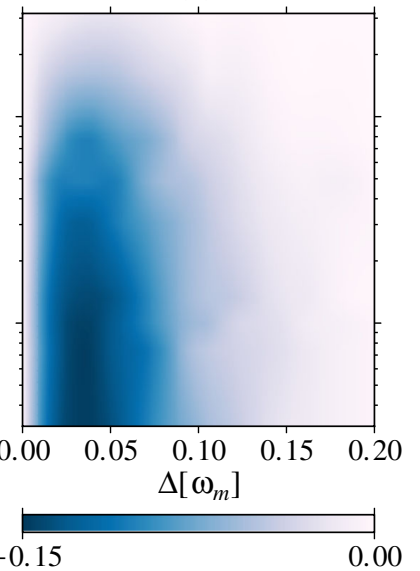

FIG. 5 (color online). (a),(b) The Fano factor $F$ versus (bare) detuning $\Delta$ and bath occupation number $\bar{n}$. Note that by varying $\bar{n}$, we automatically vary the crucial quantity $\gamma \bar{n}$ appearing in Eqs. (50) and (51). (a) Plot of the simple analytical expression (50). (b) The numerical result obtained with Monte Carlo trajectories for 30000 mechanical oscillations. (a) and (b) are in good agreement despite the fact that in the parameter regime considered here, some of the approximations are barely fulfilled. Note that the color scale in the numerical prediction for the Fano factor is slightly shifted up by 0.01 , possibly hinting at some additional diffusion process not considered in the analytical model. (c) The prediction for Wigner-function negativity (defined as the quotient of the most negative and the most positive values of $W$ ) obtained by extrapolating the results for $F$ from (b) using the function from Fig. 6. (d) The Wignerfunction negativity as directly extracted from the numerical result of the Wigner function. The constant parameters in all plots are $\left(g_{0}, \kappa_{E}=2 \kappa, \gamma_{E}=2 \gamma, E\right)=\left(0.05,0.1,10^{-7}, 1.56\right) \times \omega_{m}$. The approximate average number of photons in the cavity is 1.5 in these plots.

where $\zeta \simeq 0.27$ is the numerical value of $J_{1}^{2}$ at the position of the limit cycle. The Fano factor is minimal at an effective detuning $\Delta_{\text {eff }}\left(r_{0}\right)=\kappa$, where it takes on the value

$$
F \simeq\left(\frac{\gamma(1+2 \bar{n})}{4}+\zeta \frac{\kappa g_{0}^{2} E^{2}}{\omega_{m}^{4}}\right) /\left(\frac{\gamma}{4}+\zeta \frac{g_{0}^{2} E^{2}}{\omega_{m}^{3}}\right) .
$$

Note first that Eq. (51) implies that the Fano factor is lower bounded by the sideband resolution

$$
F>\frac{\kappa}{\omega_{m}}
$$

and that this bound is achieved for a sufficiently large driving field $E=\sqrt{2 \kappa P_{L} / \hbar \omega_{L}}$ (laser power $P_{L}$ ). Furthermore, Eq. (51) implies that the condition for sub-Poissonian statistics $1>F$ is exactly equivalent to $\left(g_{0}^{2} E^{2} / \omega_{m}^{3}\right)$ $\left(1-\frac{\kappa}{\omega_{m}}\right)>(\gamma \bar{n} / 2 \zeta)$. This can be interpreted as a condition for the driving power that for small $\kappa / \omega_{m}$ becomes

$$
\frac{P_{L}}{\hbar \omega_{L}}>\frac{\omega_{m}^{3}}{4 \zeta \kappa g_{0}^{2}} \gamma \bar{n} .
$$

It is also instructive to express this condition in terms of the (thermal, linearized) cooperativity parameter

$$
\mathcal{C}=\frac{4 g_{0}^{2} \alpha^{2}}{\kappa \gamma(2 \bar{n}+1)}=\frac{8 g_{0}^{2}}{\omega_{m}^{2} \gamma(2 \bar{n}+1)} \frac{P_{L}}{\hbar \omega_{L}},
$$

where we use that the relevant average intracavity amplitude at the optomechanical limit cycles is $\alpha=\alpha_{1} \simeq E / \omega_{m}$; cf. Eq. (28). Condition (53) then takes the form (in the limit $\bar{n} \gg 1)$

$$
\mathcal{C}>\frac{1}{\zeta} \frac{\omega_{m}}{\kappa}
$$

Note that Eq. (55) is essentially a requirement on the linearized optomechanical coupling $\left(g \propto g_{0} E\right)$ and not on the coupling per single photon $g_{0}$. The condition in Eqs. (53) and (55) and the lower bound in Eq. (52) are the main results regarding sub-Poissonian phonon statistics.

The possibility of a sub-Poissonian number distribution was discussed in Refs. [25,26] for the resonance at the first (and higher) blue sidebands. The prediction of the analytical model is especially good for the regime with small $g_{0}$ that results in larger limit-cycle amplitudes. In Fig. 5, which compares the Fano factors as derived from our analytical model and from solving the master equation, good agreement can be seen. For larger $g_{0}$ (not depicted in Fig. 5), the condition necessary for adiabatic elimination is less satisfied and also the linear approximation (49) gets worse because $\Delta n \approx\langle n\rangle$. Thus, the quantitative agreement gets worse. Still, the resonances for $F$ at $\Delta \approx 0, \omega_{m}$ are qualitatively reproduced.

In Refs. [25,26], the Fano factor has been calculated with a derivation using the truncated Wigner-function approximation and solving the resulting Langevin equation. If we use the Wigner function as the phase-space distribution, our calculation, which does not rely on this truncation, gives 
the same result in the regime where the Kerr parameter $K$ is negligible.

For limit cycles with the cavity close to its ground state, different approaches to treat the Kerr effect have been taken in the literature: Ref. [28] uses the classical part of the Kerr effect, as derived with the standard master-equation approach, to introduce a renormalized detuning with a shift proportional to the cavity occupation. An additional constant (independent of the cavity occupation) shift of the detuning by $K=g_{0}^{2} / \omega_{m}$ was numerically observed in Ref. [26] and then introduced by hand, to match the numerical data. It is one of the main results of the semipolaron approach, that the separate Kerr term for the cavity is naturally derived for limit cycles. It causes exactly the additional quantum shift of $\Delta$ observed in Ref. [26] that is most striking in the $|\alpha| \ll 1$ limit; cf. Eq. (39).

\section{E. Nonpositive Wigner function}

Finally, we use the Fano factor to predict the occurrence of a negative area in the Wigner function. For a Fock state, the Fano factor $F$ is of course zero, and, except for the vacuum, all Fock states have a pronounced negativity of the Wigner function. Both $F$ and the Wigner function are continuous functions of the state $\rho$. Hence, for a given mean phonon number $n_{0}$, there is a critical value $F_{c}$, such that for a state with $F<F_{c}$, the Wigner function has a negative area. For simple set of ansatz states given by a densitymatrix diagonal in the Fock basis with Gaussian probability distribution

$$
P(n) \propto \exp \left(-\frac{\left(n-n_{0}\right)^{2}}{V}\right)
$$

we numerically determine the corresponding critical Fano factor $F_{c}$. The result is illustrated in Fig. 6. We use this particular ansatz because the typical steady-state density matrix of our problem is approximately of this form when $g_{0} / \omega_{m}$ is not too large. In Ref. [26], the steady state as a Gaussian distribution in Fock states is derived in more detail.

Figure 6 shows that this threshold $F_{c}$ is smaller for larger amplitude $r_{0}$. We infer that in order to see the negativity of the Wigner function in the steady state, small limit-cycle amplitudes with small Fano factors are favorable. Applied to the results of Refs. [27,28], this explains the more favorable condition for negativity at the $\Delta \simeq 0$ resonance as compared to the $\Delta \simeq \omega_{m}$ resonance because the limit cycle there has a smaller amplitude [given by the first root of $J_{0}(\eta r)$ as compared to $J_{1}(\eta r)$, as discussed in Sec. IVC]. Independently of $\Delta$, the amplitude scales with the inverse of $g_{0} / \omega_{m}$, such that for a large ratio $g_{0} / \omega_{m}$, a nonpositive Wigner function is already achieved for larger Fano factors. More precisely, we can conclude from Fig. 6 that

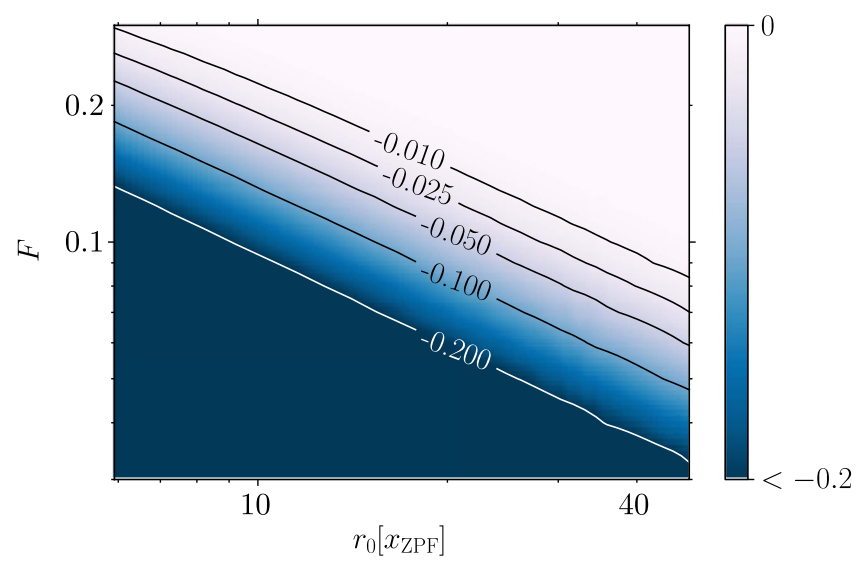

FIG. 6 (color online). Maximal negativity of the Wigner function (defined as the quotient of the most negative and the most positive values of $W$ ) as a function of the Fano factor $F$ and the mean amplitude $r_{0}$ for a phonon distribution as in Eq. (56). From this plot, one can read of how small the Fano factor needs to be for a given $r_{0}$ to see a negative value in the Wigner function. Implicitly, this is also a requirement on $g_{0}$ because $r_{0} \propto \frac{\omega_{m}}{g_{0}} ;$ see Sec. IVC.

$$
F_{c} \simeq \xi r_{0}^{-s}, \quad s \simeq 0.6,
$$

where the constant $\xi$ depends on how negative the Wigner function should be. In order to achieve a ratio of minimal to maximal values of the Wigner function of, e.g., -0.1 , this constant is found to be $\xi \simeq 0.6$. As a comparison, this negativity ratio can reach (approximately) -2.5 for odd Fock states and -0.4 for even Fock states.

Since the amplitude of the first limit cycle is $r_{0} \simeq \omega_{m} / g_{0}$, the condition $F<F_{c}$, together with Eqs. (51) and (57), is equivalent to $(\zeta \simeq 0.27)$

$\frac{g_{0}^{2} E^{2}}{\omega_{m}^{3}}\left[\xi\left(\frac{g_{0}}{\omega_{m}}\right)^{s}-\frac{\kappa}{\omega_{m}}\right]>\frac{\gamma(2 \bar{n}+1)}{4 \zeta}-\frac{\xi \gamma}{4 \zeta}\left(\frac{g_{0}}{\omega_{m}}\right)^{s}$.

Thus, one necessary condition for a negative Wigner function is that the square bracket on the left side is positive. This is a condition on the single-photon optomechanical coupling $g_{0}$ that can be written equivalently as both

$$
\frac{g_{0}}{\omega_{m}}>\left(\frac{\kappa}{\xi \omega_{m}}\right)^{1 / s}, \quad \frac{g_{0}}{\kappa}>\frac{1}{\xi^{1 / s}}\left(\frac{\kappa}{\omega_{m}}\right)^{(1-s) / s} .
$$

Note that this condition for the occurrence of a quantum state is weaker than the condition $g_{0} / \kappa>1$ that one would have naively expected.

Assuming this condition to be well fulfilled, we can drop the second terms on both the left- and right-hand sides of Eq. (57) and get the power requirement 


$$
\frac{P_{L}}{\hbar \omega_{L}}>\frac{\omega_{m}^{3}}{4 \xi \zeta \kappa g_{0}^{2}}\left(\frac{\omega_{m}}{g_{0}}\right)^{s} \gamma\left(\bar{n}+\frac{1}{2}\right)
$$

Note that Eq. (60) is stronger than the requirement (53) for sub-Poissonian statistics, as one would expect. In terms of the cooperativity (for any $\bar{n}$ ), Eq. (60) becomes

$$
\mathcal{C}>\frac{1}{\xi \zeta}\left(\frac{\omega_{m}}{g_{0}}\right)^{s} .
$$

Note also that even for zero temperature $\bar{n} \rightarrow 0$, there is now a threshold for the power (cooperativity) in contrast to the condition for sub-Poissonian statistics. Condition (59) on the strength of the optomechanical coupling per single photon and condition (60) [or (61)], which reproduces the heuristically derived condition (4) [or (5)] from Sec. II, are the main results regarding negative Wigner functions.

\section{F. Numerical analysis}

In this section, we compare the predictions from the sections above with the numerical result for the master equation of Eq. (6). To do the calculation for a large Hilbert-space dimension, we apply the Monte Carlo wavefunction method from Refs. [50-52] as implemented in QuTiP [53,54], the quantum toolbox for Python. The advantage is that one needs to simulate only wave functions and not density matrices, so that the Hilbert-space dimension required for the simulation scales only with the number of possible pure states $N$ instead of $N^{2}$. In this method, the individual trajectory of an initially pure state is calculated, conditioned on the history of fictive photon and phonon counters measuring the particles leaking out of the system. With this knowledge of the environment, an initially pure state stays pure. The density matrix is then retrieved by averaging over a large ensemble of such conditional states. The ensemble average can be replaced by the time average for calculating a steady-state density matrix.

Our implementation is done with an adaptive Hilbert space, where the Fock states are not only limited from above but also from below, and after each mechanical oscillation, the Hilbert space is updated so that it is centered around the current state. To make sure that not too much of the Hilbert space is truncated, the number of states to be used is scaled with the standard deviation in energy of the state in the previous step. This flexibility of the Hilbert space during the calculation allows us to run the simulation without much a priori knowledge of the steady state, and even fewer basis states are required.

The solution is obtained in the following steps: For a speedup of the calculation, the initial state is chosen to be a coherent state with an amplitude close to the expected steady state. It is then evolved for some period until, at a time $t_{0}$, the conditional state's amplitude and Fano factor stop to drift and only fluctuate. We then make use of the fact that in the steady state, the time average corresponds to the ensemble average, and calculate the steady state of the oscillator as

$$
\rho_{M}=\int_{t_{0}}^{t_{0}+T} \operatorname{tr}_{c}\left(\left|\psi_{t}\right\rangle\left\langle\psi_{t}\right|\right) d t
$$

where $\left|\psi_{t}\right\rangle$ is the conditional state at time $t$ and $T$ spans many mechanical oscillations.

This procedure is performed many times in parallel on a cluster, and the resulting matrices $\rho_{M}$ are averaged. The deviation of the individual $\rho_{M}$ provides an error estimate for the method. As a further benchmark and control, we also calculate the steady state with the biconjugate-gradient steady-state solver from SciPy [55] that is, however, limited to a comparably small Hilbert-space dimension.

The algorithm described above allows us for the first time to numerically study optomechanical limit cycles in the experimentally relevant regime of large amplitudes of the mechanical oscillator (as caused by a relatively small $\left.g_{0} / \omega_{m}\right)$ and with more than only a few photons in the cavity. In previous studies, the question was posed as to whether the analytical theory can be applied to this regime [26] and if the nonclassical features survive [28] for more than one photon in the cavity. We answer this question in the affirmative: Fig. 7 shows an example of a Wigner function in this regime with a small Fano factor and some negative density.

Strictly speaking, the steady state calculated here is only metastable if $\gamma$ is so small that there is more than one attractor for the limit cycle; cf. Fig. 1. The time scale for switching between different attractors is much longer than the time to relax in a given metastable steady state. Thus, it is not considered in this article. In order to choose the metastable attractor for the numerical simulation, we choose an initial state in the vicinity of our preferred attractor, in this case, the

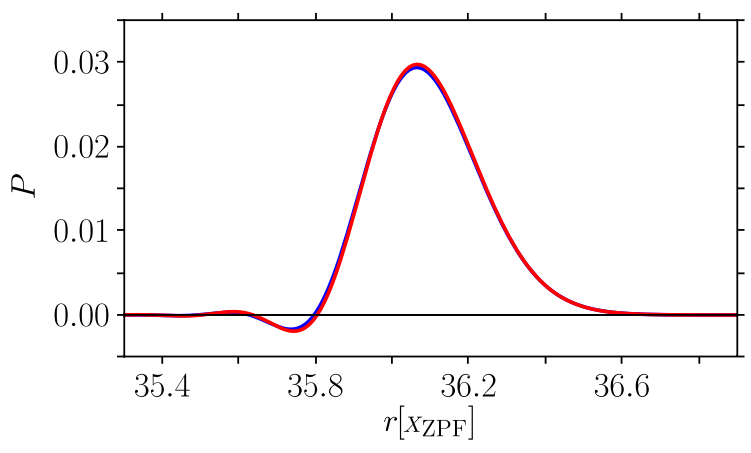

FIG. 7 (color online). Radial part of a Wigner function for parameters with high amplitude and many photons $\left(\left\langle a^{\dagger} a\right\rangle \approx 8\right)$ in the cavity featuring a very small Fano factor $(F=0.07)$ and some negative density. The parameters are $\left(g_{0}, \kappa_{E}=\right.$ $2 \kappa, \gamma, E, \Delta)=(0.033,0.1,0,3.5,0.03) \times \omega_{m}$. The blue and red lines are the results of two independent runs (each averaging 5000 mechanical oscillations) of the Monte Carlo-based steady-state solver. 
limit cycle with the lowest possible amplitude. Also, in the analytical expressions for the Fano factor, we always treat possibly metastable states as steady states. For very large $g_{0} / \omega_{m}$, different metastable attractors start to merge and the analysis becomes more involved. This merging of attractors and its effect on nonclassical features was studied in detail in Ref. [28].

\section{CONCLUSIONS}

We studied the quantum regime of optomechanical limit cycles. Based on the laser theory of Haake and Lewenstein [31], we derived an effective Fokker-Planck equation for an optomechanical system. The analytical prediction for the oscillator's steady state is in agreement with the work of Rodrigues and Armour [25,26] for driving fields on the first blue sideband.

Our treatment naturally also includes the Kerr effect, which becomes important for large $g_{0}^{2} / \omega_{m}$. One consequence important for the quantum theory of limit cycles is the shift of the detuning of Eq. (39) that occurs even without photons in the cavity and had to be introduced phenomenologically in Ref. [26]. This shift explains the possibility of limit cycles on the blue sideband in Ref. [27] or for the parameters of Fig. 2.

The effective cavity detuning is usually approximated as a static variable. Within our framework, one can describe its dynamical nature, which is a classical phenomenon scaling proportional to the Kerr parameter. Figures 4 and 3 show how this smoothens the phase transition between optomechanical cooling and self-induced oscillations.

We studied the quantum limit cycles on resonance and found the simple analytical expression (50) that predicts the possibility of very small values for the Fano factor $F$ of the mechanical oscillator. We found that in the sideband-resolved regime, a large value of $\left(g_{0}^{2} E^{2} / \omega_{m}^{3} \gamma \bar{n}\right)$, i.e., a large linearized optomechanical coupling, is required to minimize $F$.

We then established a relation between sub-Poissonian phonon statistics and negativity of the Wigner density for typical parameters of limit cycles: The oscillator's steady state has an approximately Gaussian number distribution at each metastable limit cycle. For these states, the requirement on $F$ to see the negativity of the Wigner function is given by the function of Fig. 6 .

Using a Monte Carlo method with an adaptive Hilbert space, we numerically checked this scaling even for limit cycles with very large amplitude and many photons in the cavity, where an ordinary steady-state solver cannot be applied. The numerical simulation depicted in Fig. 5 shows that, indeed, the criterion of a small Fano factor can predict the negativity of the Wigner function. For currently more feasible experimental parameters with even smaller $g_{0} / \omega_{m}$, the negativity disappears according to Fig. 6 but the very small Fano factors remain.

We believe that the present approach provides a suitable starting point for further studies of optomechanical systems in the limit of strong couplings. We point out once more that in the "semipolaron picture" introduced here, the Kerr nonlinearity and the optomechanical interaction occur as independent terms. This enables us, in principle, to take into account the squeezed noise of the cavity when deriving effective equations of motion of the mechanical oscillator. black While for the parameters considered in this article we could neglect this effect, additional diffusion for the mechanical oscillator is to be expected for a very strong laser drive. This would apply to the case of limit cycles but could also become important in the cooling regime.

\section{ACKNOWLEDGMENTS}

This work was funded by the Centre for Quantum Engineering and Space-Time Research (QUEST) at the Leibniz University of Hannover and by the European Community (FP7-Programme) through iQUOEMS (Grant Agreement No. 323924). We acknowledge the support of the cluster system team at the Leibniz University of Hannover in the production of this work. We thank Denis Vasilyev and Kai Stannigl for fruitful discussions.

\section{APPENDIX A: TRANSFORMATIONS FOR GENERAL PHASE-SPACE DISTRIBUTION}

\section{Semipolaron transformation}

In the main text, we introduced the semipolaron transformation only for the special case of the $Q$ function, to make the equations more readable. Here, we drop this restriction and assume the more general case of an $s$-parametrized phase-space distribution $P_{s}$ with $s \in[-1,1]$. For the convenience of the calculation, we define $p=\frac{s+1}{2} \in[0,1]$ and $q=1-p$. Note that for $q=0$, this definition corresponds to the Glauber-Sudarshan $P$ representation, for $q=\frac{1}{2}$ to the Wigner representation, and for $q=1$ to the Husimi $Q$ representation.

Starting from the standard optomechanical Hamiltonian and Lindblad operators, we first switch to a displaced and rotating frame with frequency $\omega_{m}$ for the mechanical oscillator so that $b \rightarrow \beta_{0}+b e^{-i \omega_{m} t}$ and introduce the shorthand notation $b_{t}=b e^{-i \omega_{m} t}$. This transformation also leaves the Lindblad operators unchanged, and the Hamiltonian transforms to

$$
\begin{aligned}
H= & \left(\omega_{m}+i \gamma\right) \beta_{0}^{*} b_{t}+\left(\omega_{m}-i \gamma\right) \beta_{0} b_{t}^{\dagger}-g_{0}\left(\beta_{0}+\beta_{0}^{*}\right) a^{\dagger} a \\
& -\Delta a^{\dagger} a-g_{0} a^{\dagger} a\left(b_{t}+b_{t}^{\dagger}\right)-i E\left(a-a^{\dagger}\right) .
\end{aligned}
$$

Using the translation rules

$$
b \rho \rightarrow\left(\beta+q \partial_{\beta^{*}}\right) \sigma, \quad b^{\dagger} \rho \rightarrow\left(\beta^{*}-p \partial_{\beta}\right) \sigma,
$$

we obtain the translated equation of motion $\dot{\sigma}\left(\beta, \beta^{*}\right)=$ $L_{c} \sigma+L_{m} \sigma+L_{\text {int }} \sigma$. With the shorthands $\beta_{t}=\beta e^{-i \omega_{m} t}$ and $\partial_{\beta_{t}}=\partial_{\beta} e^{i \omega_{m} t}$, this gives 


$$
\begin{aligned}
\begin{aligned}
L_{c} \sigma= & -i\left[-g_{0}\left(\beta_{0}+\beta_{0}^{*}\right) a^{\dagger} a-\Delta a^{\dagger} a-g_{0} a^{\dagger} a\left(\beta_{t}+\beta_{t}^{*}\right), \sigma\right] \\
& -i\left[-i E\left(a-a^{\dagger}\right), \sigma\right]+L_{c} \sigma, \\
L_{\mathrm{int}} \sigma= & \text { (A2) } g_{0}\left[\left(q \partial_{\beta_{t}}-p \partial_{\beta_{t}^{*}}\right) \sigma a^{\dagger} a-\left(q \partial_{\beta_{t}^{*}}-p \partial_{\beta_{t}}\right) a^{\dagger} a \sigma\right], \\
L_{m} \sigma= & -i\left(\omega_{m}+i \gamma\right) \beta_{0}^{*} \partial_{\beta_{t}^{*}} \sigma+i\left(\omega_{m}-i \gamma\right) \beta_{0} \partial_{\beta_{t}} \sigma+I_{m} \sigma,
\end{aligned}
\end{aligned}
$$

with

$$
\begin{gathered}
I_{m}=\gamma\left(\partial_{\beta} \beta+\partial_{\beta^{*}} \beta^{*}\right)+2 \gamma\left(\bar{n}+q^{2}+p q\right) \partial_{\beta^{*}} \partial_{\beta}, \\
L_{c}=\kappa D[a] .
\end{gathered}
$$

In analogy to transformation (15), we apply the more general

$$
\tilde{\sigma}(t)=\exp \left[-i \theta(t) a^{\dagger} a\right] \sigma(t) \exp \left[i \theta(t) a^{\dagger} a\right],
$$

with parameters $\lambda=\lambda_{r}+i \lambda_{i}=\frac{g_{0}}{\omega_{m}+i \gamma} \quad$ and $\quad \theta(t)=$ $i\left(\lambda \beta e^{-i \omega_{m} t}-\lambda^{*} \beta^{*} e^{i \omega_{m} t}\right)$, which gives

$$
\begin{aligned}
L_{c} \sigma= & -i\left[-\Delta a^{\dagger} a-g_{0} \lambda_{r}\left(a^{\dagger} a\right)^{2}\right. \\
& \left.-i E\left(e^{i \theta(t)} a-e^{-i \theta(t)} a^{\dagger}\right), \sigma\right]+L_{c} \sigma \\
& +2 \gamma|\lambda|^{2}\left(\bar{n}+q^{2}+p q+\frac{q-p}{2}\right) D\left[a^{\dagger} a\right] \sigma,
\end{aligned}
$$

$$
\begin{aligned}
L_{\text {int }} \sigma= & -i g_{0}\left[\left(q \partial_{\beta_{t}}-p \partial_{\beta_{t}^{*}}\right) \sigma a^{\dagger} a-\left(q \partial_{\beta_{t}^{*}}-p \partial_{\beta_{t}}\right) a^{\dagger} a \sigma\right] \\
& +2 \gamma\left(\bar{n}+q^{2}+p q\right)\left\{\left(\lambda^{*} \partial_{\beta_{t}}-\lambda \partial_{\beta_{t}^{*}}\right)\left[a^{\dagger} a, \sigma\right]\right\},
\end{aligned}
$$

$$
L_{m} \sigma=I_{m} \sigma-i\left(\omega_{m}+i \gamma\right) \beta_{0}^{*} \partial_{\beta_{t}^{*}} \sigma+i\left(\omega_{m}-i \gamma\right) \beta_{0} \partial_{\beta_{t}} \sigma
$$

and includes terms of order $\frac{1}{Q}$. After dropping these terms as an approximation, this is, with $K=\frac{g_{0}^{2}}{\omega_{m}}$,

$$
\begin{aligned}
L_{c} \sigma= & -i\left[-\Delta a^{\dagger} a-K\left(a^{\dagger} a\right)^{2}-i E\left(e^{i \theta(t)} a-e^{-i \theta(t)} a^{\dagger}\right), \sigma\right] \\
& +L_{c} \sigma,
\end{aligned}
$$

$$
L_{\text {int }} \sigma=-i g_{0}\left[\left(q \partial_{\beta_{t}}-p \partial_{\beta_{t}^{*}}\right) \sigma a^{\dagger} a-\left(q \partial_{\beta_{t}^{*}}-p \partial_{\beta_{t}}\right) a^{\dagger} a \sigma\right],
$$

$$
L_{m} \sigma=I_{m} \sigma-i\left(\omega_{m}+i \gamma\right) \beta_{0}^{*} \partial_{\beta_{t}^{*}} \sigma+i\left(\omega_{m}-i \gamma\right) \beta_{0} \partial_{\beta_{t}} \sigma .
$$

We now transform to a displaced frame $\tilde{\sigma}=$ $D^{\dagger}(\alpha) \sigma D(\alpha)$ with parameter $\alpha(\beta, t) \in \mathbb{C}$. For a master equation of the form

$$
\dot{\rho}=-i\left\{-\Delta a^{\dagger} a-K\left(a^{\dagger} a\right)^{2}-i\left[E(t) a-E^{*}(t) a^{\dagger}\right], \rho\right\}+L_{c} \rho,
$$

the transformation to a displaced frame $\tilde{\rho}=$ $D^{\dagger}[\alpha(t)] \rho D[\alpha(t)]$ gives

$$
\begin{aligned}
\dot{\tilde{\rho}}= & L \tilde{\rho} \\
& -i\left\{-\left(\Delta+4 K|\alpha|^{2}\right) a^{\dagger} a-K\left(a^{\dagger} a\right)^{2}\right. \\
& -K\left[\alpha^{2}\left(a^{\dagger}\right)^{2}+\left(\alpha^{*} a+\alpha a^{\dagger}\right) a^{\dagger} a+\text { H.c. }\right] \\
& \left.-i\left[\left(\dot{\alpha}+\left(\kappa-i \Delta-i 2 K|\alpha|^{2}\right) \alpha-E\right) a^{\dagger}-\text { H.c. }\right], \tilde{\rho}\right\} .
\end{aligned}
$$

Depending on whether one wants to study the regime $|\alpha| \gg 1$ or $|\alpha| \ll 1$, either the terms with low or high order in $\alpha$ can be neglected at this point and a different choice of $\alpha(t)$ is required to cancel all displacementlike terms.

\section{Displaced frame for $|\alpha| \gg 1$}

We can cancel the displacementlike terms, which include the terms of order $K|\alpha|^{3}$, by imposing that $\alpha(t)$ solves

$$
\dot{\alpha}(t)=\left\{i\left[\Delta+2 K|\alpha(t)|^{2}\right]-\kappa\right\} \alpha(t)+E(t),
$$

such that in the displaced frame,

$$
\begin{aligned}
\dot{\tilde{\rho}}= & -i\left\{-\left(\Delta+4 K|\alpha|^{2}\right) a^{\dagger} a-K\left[\alpha^{2}\left(a^{\dagger}\right)^{2}\right.\right. \\
& \left.\left.+\left(\alpha^{*} a+\alpha a^{\dagger}\right) a^{\dagger} a+\text { H.c. }\right]-K\left(a^{\dagger} a\right)^{2}, \tilde{\rho}\right\}+L \tilde{\rho}
\end{aligned}
$$

Neglecting the terms proportional to $K$ up to first order in $\alpha$, the Liouvillians are

$$
\begin{aligned}
& L_{c} \sigma=-i\left\{-\left(\Delta+4 K|\alpha|^{2}\right) a^{\dagger} a-K\left[\alpha^{2}\left(a^{\dagger}\right)^{2}+\text { H.c. }\right], \sigma\right\} \\
& +L_{c} \sigma, \\
& \begin{aligned}
L_{\mathrm{int}} \sigma= & -i g_{0}\left(q \partial_{\beta_{t}}-p \partial_{\beta_{t}^{*}}\right) \sigma\left(\alpha^{*} a+\alpha a^{\dagger}\right) \\
& +i g_{0}\left(q \partial_{\beta_{t}^{*}}-p \partial_{\beta_{t}}\right)\left(\alpha^{*} a+\alpha a^{\dagger}\right) \sigma, \\
L_{m} \sigma= & -i g_{0}\left(\partial_{\beta_{t}}-\partial_{\beta_{t}^{*}}\right)|\alpha|^{2} \sigma+I_{m} \sigma-i\left(\omega_{m}+i \gamma\right) \beta_{0}^{*} \partial_{\beta_{t}^{*}} \sigma \\
& +i\left(\omega_{m}-i \gamma\right) \beta_{0} \partial_{\beta_{t}} \sigma .
\end{aligned}
\end{aligned}
$$


Note that now, in analogy to laser theory, $L_{m}$ reproduces the classical drift. We have now $\left|L_{\text {int }}\right| \propto g_{0}|\alpha| \ll$ $g_{0}\left\langle a^{\dagger} a\right\rangle \approx g_{0}|\alpha|^{2}$, where $\left\langle a^{\dagger} a\right\rangle$ refers to the average before the transformation.

\section{Displaced frame for $|\alpha| \ll 1$}

If we restrict the analysis to only the lowest two Fock states, the operators consisting of three creation or annihilation operators resulting from transformation (A13) can be approximated with just one operator, e.g., $a a^{\dagger} a \approx a$. This time, we neglect the terms proportional to $K$ of third order in $\alpha$. By imposing that $\alpha(t)$ solves this time

$$
\dot{\alpha}(t)=[i(\Delta+K)-\kappa] \alpha(t)+E(t),
$$

we can cancel the remaining displacementlike terms. The Liouvillians are now

$$
\begin{aligned}
L_{c} \sigma=- & i\left\{-\Delta a^{\dagger} a-K\left(a^{\dagger} a\right)^{2}-K\left[\alpha^{2}\left(a^{\dagger}\right)^{2}+\text { H.c. }\right], \sigma\right\} \\
& +L_{c} \sigma, \\
L_{\text {int }} \sigma= & -i g_{0}\left(q \partial_{\beta_{t}}-p \partial_{\beta_{t}^{*}}\right) \sigma\left(\alpha^{*} a+\alpha a^{\dagger}+a^{\dagger} a\right) \\
& +i g_{0}\left(q \partial_{\beta_{t}^{*}}-p \partial_{\beta_{t}}\right)\left(\alpha^{*} a+\alpha a^{\dagger}+a^{\dagger} a\right) \sigma, \quad \text { (A20) } \\
L_{m} \sigma=- & i g_{0}\left(\partial_{\beta_{t}}-\partial_{\beta_{t}^{*}}\right)|\alpha|^{2} \sigma+I_{m} \sigma-i\left(\omega_{m}+i \gamma\right) \beta_{0}^{*} \partial_{\beta_{t}^{*}} \sigma \\
& +i\left(\omega_{m}-i \gamma\right) \beta_{0} \partial_{\beta_{t}} \sigma .
\end{aligned}
$$

\section{APPENDIX B: DERIVATION OF THE FOKKER-PLANCK EQUATION}

In order to obtain the approximate Fokker-Planck equation for the mechanical oscillator, we now eliminate the cavity in second-order perturbation theory. We show this calculation in detail for $|\alpha| \gg 1$ and then briefly write down the results for $|\alpha| \ll 1$.

\section{Adiabatic elimination of the cavity in the $|\alpha| \gg 1$ regime}

Let us for now ignore $\mathcal{L}_{m}$ and reinclude it later. Defining $\sigma_{i j}:=\langle i|\sigma| j\rangle$ and cutting off after the index $(i, j)=(1,1)$, we get the equation of motion (EOM)

$$
\begin{aligned}
\dot{\sigma}_{00}= & 2 \kappa \sigma_{11}+i g_{0}\left[\left(q \partial_{\beta_{t}^{*}}-p \partial_{\beta_{t}}\right) \alpha_{t}^{*} \sigma_{10}-\left(q \partial_{\beta_{t}}-p \partial_{\beta_{t}^{*}}\right) \alpha_{t} \sigma_{01}\right] \\
& +i g_{0}\left(\partial_{\beta_{t}^{*}} \alpha_{t}^{*} \alpha_{t} \sigma_{00}-\partial_{\beta_{t}} \alpha_{t}^{*} \alpha_{t} \sigma_{00}\right), \\
\dot{\sigma}_{11}= & -2 \kappa \sigma_{11}+i g_{0}\left[\left(q \partial_{\beta_{t}^{*}}-p \partial_{\beta_{t}}\right) \alpha_{t} \sigma_{01}-\left(q \partial_{\beta_{t}}-p \partial_{\beta_{t}^{*}}\right) \alpha_{t}^{*} \sigma_{10}\right] \\
& +i g_{0}\left(\partial_{\beta_{t}^{*}} \sigma_{11}-\partial_{\beta_{t}} \sigma_{11}\right)+i g_{0}\left(\partial_{\beta_{t}^{*}} \alpha_{t}^{*} \alpha_{t} \sigma_{11}-\partial_{\beta_{t}} \alpha_{t}^{*} \alpha_{t} \sigma_{11}\right),
\end{aligned}
$$

$$
\begin{aligned}
\dot{\sigma}_{10}= & -\kappa \sigma_{10}+i \tilde{\Delta}_{\mathrm{eff}} \sigma_{10}+i g_{0} \partial_{\beta_{t}^{*}} \sigma_{10} \\
& +i g_{0}\left[\left(q \partial_{\beta_{t}^{*}}-p \partial_{\beta_{t}}\right) \alpha_{t} \sigma_{00}-\left(q \partial_{\beta_{t}}-p \partial_{\beta_{t}^{*}}\right) \alpha_{t} \sigma_{11}\right] \\
& +i g_{0}\left(\partial_{\beta_{t}^{*}} \alpha_{t}^{*} \alpha_{t} \sigma_{10}-\partial_{\beta_{t}} \alpha_{t}^{*} \alpha_{t} \sigma_{10}\right) .
\end{aligned}
$$

We now adiabatically eliminate $\sigma_{10}$ to first order in $g_{0}$ (note that $\sigma_{11}$ is already of order $g_{0}^{2}$ ):

$\sigma_{10}(t)=\int_{0}^{\infty} d \tau e^{-\kappa \tau+i \tilde{\Delta}_{\mathrm{eff}} \tau} i g_{0}\left(q \partial_{\beta_{t-\tau}^{*}}-p \partial_{\beta_{t-\tau}}\right) \alpha_{t-\tau} \sigma_{00}(t)$

$$
\begin{aligned}
= & i g_{0} \sum_{n}\left(q \partial_{\beta^{*}} e^{i(n-1) \omega_{M} t} \frac{\alpha_{n}}{\tilde{h}_{n-1}}-p \partial_{\beta} e^{i(n+1) \omega_{M} t} \frac{\alpha_{n}}{\tilde{h}_{n+1}}\right) \\
& \times \sigma_{00}(t),
\end{aligned}
$$

where $h_{n}=\kappa+i\left(n \omega_{M}-\tilde{\Delta}_{\text {eff }}\right)$ and $\alpha=\sum_{n=-\infty}^{\infty} \alpha_{n} e^{\text {in } \omega_{m} t}$, with $\alpha_{n}=\Xi_{n} e^{- \text {in } \phi}$. Now, the derivative of the phasespace distribution is approximately given by $\dot{P}_{s}\left(\beta, \beta^{*}\right) \approx$ $\operatorname{Tr}\left[\dot{\sigma}_{00}\left(\beta, \beta^{*}\right)+\dot{\sigma}_{11}\left(\beta, \beta^{*}\right)\right]$, which gives to second order in $g_{0}$

$$
\begin{aligned}
\dot{P}_{s}= & \sum_{n} q^{2} g_{0}^{2}\left(\partial_{\beta^{*}} \partial_{\beta} \frac{\alpha_{n}^{*} \alpha_{n}}{\tilde{h}_{n-1}}-\partial_{\beta^{*}} \partial_{\beta^{*}} \frac{\alpha_{n-2}^{*} \alpha_{n}}{\tilde{h}_{n-1}}\right) P_{s} \\
& +p^{2} g_{0}^{2}\left(\partial_{\beta^{*}} \partial_{\beta} \frac{\alpha_{n}^{*} \alpha_{n}}{\tilde{h}_{n+1}}-\partial_{\beta} \partial_{\beta} \frac{\alpha_{n+2}^{*} \alpha_{n}}{\tilde{h}_{n+1}}\right) P_{s} \\
& +p q g_{0}^{2}\left[\partial_{\beta^{*}} \partial_{\beta}\left(\frac{\alpha_{n}^{*} \alpha_{n}}{\tilde{h}_{n+1}}+\frac{\alpha_{n}^{*} \alpha_{n}}{\tilde{h}_{n-1}}\right)-\partial_{\beta}^{2} \frac{\alpha_{n+2}^{*} \alpha_{n}}{\tilde{h}_{n+1}}\right. \\
& \left.-\partial_{\beta^{*}}^{2} \frac{\alpha_{n-2}^{*} \alpha_{n}}{\tilde{h}_{n-1}}\right] P_{s}+i g_{0}\left(\partial_{\beta^{*}} \alpha_{n-1}^{*} \alpha_{n}\right) P_{s}+\text { H.c., }
\end{aligned}
$$

where we neglect terms $\propto \frac{1}{r}$, as they are negligible at the position of the limit cycle. Note that the drift term does not depend on the choice of phase-space distribution. For the $Q$ function, the equation simplifies to

$$
\begin{aligned}
\dot{Q} & =g_{0}^{2} \sum_{n}\left(\partial_{\beta^{*}} \partial_{\beta} \frac{2 \kappa \alpha_{n}^{*} \alpha_{n}}{\left|\tilde{h}_{n-1}\right|^{2}}-\partial_{\beta^{*}} \partial_{\beta^{*}} \frac{\alpha_{n-2}^{*} \alpha_{n}}{\tilde{h}_{n-1}}-\partial_{\beta} \partial_{\beta} \frac{\alpha_{n-2} \alpha_{n}^{*}}{\tilde{h}_{n-1}^{*}}\right) Q \\
& +i g_{0} \sum_{n}\left(\partial_{\beta^{*}} \alpha_{n-1}^{*} \alpha_{n}-\partial_{\beta} \alpha_{n-1} \alpha_{n}^{*}\right) Q
\end{aligned}
$$

and for the Wigner function to

$$
\begin{aligned}
\dot{W}= & \sum_{n} \frac{g_{0}^{2} \kappa}{\left|\tilde{h}_{n+1}\right|^{2}}\left[\partial_{\beta^{*}} \partial_{\beta}\left(\left|\alpha_{n}\right|^{2}+\left|\alpha_{n+2}\right|^{2}\right)\right. \\
& \left.-\partial_{\beta}^{2} \alpha_{n+2}^{*} \alpha_{n}-\partial_{\beta^{*}}^{2} \alpha_{n}^{*} \alpha_{n+2}\right] W \\
& +i g_{0} \sum_{n}\left(\partial_{\beta^{*}} \alpha_{n-1}^{*} \alpha_{n}-\partial_{\beta} \alpha_{n-1} \alpha_{n}^{*}\right) W .
\end{aligned}
$$




\section{Transformation to polar coordinates}

We are finally interested in the EOM for polar coordinates $(r, \phi)$. When previously $\int d \alpha d \alpha^{*} P_{s}\left(\alpha, \alpha^{*}\right)=1$, the new normalization is $\int r d r d \varphi P_{s}(r, \phi)=1$. With

$$
\begin{gathered}
\partial_{x}=\cos (\phi) \partial_{r}-\frac{\sin (\phi)}{r} \partial_{\phi}, \\
\partial_{y}=\sin (\phi) \partial_{r}+\frac{\cos (\phi)}{r} \partial_{\phi},
\end{gathered}
$$

we get

$$
\begin{gathered}
\partial_{\beta}=\frac{1}{2} e^{-i \phi}\left(\partial_{r}-\frac{i}{r} \partial_{\phi}\right), \\
\partial_{\beta^{*}}=\frac{1}{2} e^{i \phi}\left(\partial_{r}+\frac{i}{r} \partial_{\phi}\right)
\end{gathered}
$$

and

$$
\begin{gathered}
\left(2 \partial_{\beta}\right)^{2}=e^{-2 i \phi}\left(\partial_{r}^{2}-2 \frac{i}{r} \partial_{r \phi}+2 \frac{i}{r^{2}} \partial_{\phi}-\frac{1}{r} \partial_{r}-\frac{1}{r^{2}} \partial_{\phi}^{2}\right), \\
\left(2 \partial_{\beta}\right)\left(2 \partial_{\beta}^{*}\right)=\partial_{r}^{2}+\frac{1}{r} \partial_{r}+\frac{1}{r^{2}} \partial_{\phi}^{2} .
\end{gathered}
$$

Integrating out $\phi$ and again neglecting terms $\propto \frac{1}{r}$, we get, e.g., for the $Q$ distribution,

$$
\begin{aligned}
\dot{Q}= & \sum_{n} \frac{g_{0}^{2}}{2} \partial_{r}^{2}\left(\frac{\kappa \Xi_{n}^{*} \Xi_{n}}{\left|\tilde{h}_{n-1}\right|^{2}}-\operatorname{Re}\left[\frac{\Xi_{n-2}^{*} \Xi_{n}}{\tilde{h}_{n-1}}\right]\right) Q \\
& +g_{0} \partial_{r}\left(\operatorname{Im}\left[\Xi_{n-1} \Xi_{n}^{*}\right]\right) Q \\
& -\frac{1}{r} \partial_{r} \frac{g_{0}^{2}}{2}\left(\frac{\kappa \Xi_{n}^{*} \Xi_{n}}{\left|\tilde{h}_{n-1}\right|^{2}}-\operatorname{Re}\left[\frac{\Xi_{n-2}^{*} \Xi_{n}}{\tilde{h}_{n-1}}\right]\right) Q
\end{aligned}
$$

or, in compact form with $J_{n}:=J_{n}(-\eta r)$ and including $\mathcal{L}_{m}$, this gives the parameters

$$
\begin{aligned}
D_{Q}= & \frac{\gamma(1+\bar{n})}{2} \\
& +\sum_{n} \frac{g_{0}^{2} E^{2}}{2}\left(\frac{\kappa J_{n} J_{n}}{\left|h_{n}\right|^{2}\left|\tilde{h}_{n-1}\right|^{2}}-\operatorname{Re}\left[\frac{J_{n-2} J_{n}}{\tilde{h}_{n-1} h_{n-2}^{*} h_{n}}\right]\right) \\
& \mu_{Q}=-\gamma r-\sum_{n} g_{0} E^{2}\left(\operatorname{Im}\left[\frac{J_{n-1} J_{n}}{h_{n-1} h_{n}^{*}}\right]\right)
\end{aligned}
$$

for the FPE

$$
\dot{P}_{s}=-\partial_{r} \mu_{s} P_{s}+\partial_{r}^{2} D_{s} P_{s}
$$

that can be solved (up to normalization) as

$$
P_{s}(r) \propto \frac{e^{I_{s}(r)}}{D_{s}(r)}, \quad I_{s}(r):=\int_{0}^{r} \frac{\mu_{s}\left(r^{\prime}\right)}{D_{s}\left(r^{\prime}\right)} d r^{\prime} .
$$

The corresponding equation for the Wigner function has the same drift coefficient and a diffusion of

$$
\begin{aligned}
D_{W}(r)= & \frac{\gamma(2 \bar{n}+1)}{4}+\sum_{n} \frac{\kappa g_{0}^{2} E^{2}}{4\left|\tilde{h}_{n+1}\right|^{2}}\left(\left|\frac{J_{n+2}}{h_{n+2}}\right|^{2}\right. \\
& \left.+\left|\frac{J_{n}}{h_{n}}\right|^{2}-\frac{J_{n} J_{n+2}}{h_{n} h_{n+2}^{*}}-\frac{J_{n} J_{n+2}}{h_{n}^{*} h_{n+2}}\right) .
\end{aligned}
$$

In both cases, we assume in the steady state that $P_{s}(r, \phi)=P_{s}(r)$; i.e., the distributions are independent of $\phi$.

\section{Fokker-Planck equation for $|\alpha|^{2} \ll 1$}

The procedure of the adiabatic elimination is in complete analogy to $|\alpha|^{2} \gg 1$. One only has to replace $\Delta_{\text {eff }}$ and $\tilde{\Delta}_{\text {eff }}$ with $\Delta_{K}=\Delta+K$ and adjust the solution of $\alpha$ as in Eq. (A18). With $h_{n}=\kappa+i\left(n \omega_{m}-\Delta_{K}\right)$, the final coefficients for the Fokker-Planck equation then have the same structure but without the distinction between $h_{n}$ and $\tilde{h}_{n}$; e.g., for the $Q$ function, one obtains

$$
\begin{aligned}
D_{Q}= & \frac{\gamma(1+\bar{n})}{2} \\
& +\sum_{n} \frac{g_{0}^{2} E^{2}}{2}\left(\frac{\kappa J_{n} J_{n}}{\left|h_{n}\right|^{2}\left|h_{n-1}\right|^{2}}-\operatorname{Re}\left[\frac{J_{n-2} J_{n}}{h_{n-1} h_{n-2}^{*} h_{n}}\right]\right) \\
& \mu_{Q}=-\gamma r-\sum_{n} g E^{2}\left(\operatorname{Im}\left[\frac{J_{n-1} J_{n}}{h_{n-1} h_{n}^{*}}\right]\right)
\end{aligned}
$$

\section{APPENDIX C: SEMIPOLARON TRANSFORMATION}

The semipolaron transformation [Eq. (15) in Sec. III] is introduced in terms of the formalism of quasiprobability distributions. In view of the similarities of this transformation with the polaron transformation in Eq. (19), the question arises as to how the semipolaron transformation in Eq. (15) can be expressed in terms of an ordinary operator representation. The transformed state $\tilde{\sigma}$ in Eq. (15) fulfills 


$$
\partial_{\eta} \tilde{\sigma}=\left[\frac{1}{2}\left(\beta-\beta^{*}\right) a^{\dagger} a, \tilde{\sigma}\right]=\frac{1}{2}\left[a^{\dagger} a, \tilde{\sigma} \beta-\beta^{*} \tilde{\sigma}\right]
$$

When written in the second form, we can apply the replacement rules (11) to write the last equation in operator representation

$$
\begin{aligned}
\partial_{\eta} \tilde{\rho}= & \frac{1}{2}\left[a^{\dagger} a, \tilde{\rho} b-b^{\dagger} \tilde{\rho}\right] \\
= & \frac{1}{4}\left[\left(b-b^{\dagger}\right) a^{\dagger} a, \tilde{\rho}\right] \\
& +\frac{1}{4}\left(D\left[b^{\dagger}+a^{\dagger} a\right]-D\left[b^{\dagger}\right]-D\left[a^{\dagger} a\right]\right) \tilde{\rho} \\
\equiv & L_{\text {semipol }} \tilde{\rho} .
\end{aligned}
$$

In the second line, we express the generator for the semipolaron transformation in terms of a commutator with a Hamiltonian and three Lindblad terms. The semipolaron transformation in operator representation is thus

$$
\tilde{\rho}=\exp \left(\eta L_{\text {semipol }}\right) \rho .
$$

It becomes equivalent to the polaron transformation if the Lindblad terms in the generator $L_{\text {semipol }}$ are dropped. Thus, the semipolaron transformation is nonunitary. In the context of adiabatic elimination of a cavity mode in the bad cavity limit, a similar transformation to a "dissipation picture" was employed in Refs. [56,57].

[1] M. Aspelmeyer, T. J. Kippenberg, and F. Marquardt, Cavity Optomechanics, arXiv:1303.0733.

[2] P. Meystre, A Short Walk through Quantum Optomechanics, Ann. Phys. (Berlin) 525, 215 (2013).

[3] Y. Chen, Macroscopic Quantum Mechanics: Theory and Experimental Concepts of Optomechanics, J. Phys. B 46, 104001 (2013).

[4] J. D. Teufel, T. Donner, D. Li, J. W. Harlow, M. S. Allman, K. Cicak, A. J. Sirois, J. D. Whittaker, K. W. Lehnert, and R. W. Simmonds, Sideband Cooling of Micromechanical Motion to the Quantum Ground State, Nature (London) 475, 359 (2011).

[5] J. Chan, T. P. M. Alegre, A. H. Safavi-Naeini, J. T. Hill, A. Krause, S. Gröblacher, M. Aspelmeyer, and O. Painter, Laser Cooling of a Nanomechanical Oscillator into Its Quantum Ground State, Nature (London) 478, 89 (2011).

[6] D. W. C. Brooks, T. Botter, S. Schreppler, T. P. Purdy, N. Brahms, and D. M. Stamper-Kurn, Non-classical Light Generated by Quantum-Noise-Driven Cavity Optomechanics, Nature (London) 488, 476 (2012).

[7] A. H. Safavi-Naeini, S. Groeblacher, J. T. Hill, J. Chan, M. Aspelmeyer, and O. Painter, Squeezing of Light via Reflection from a Silicon Micromechanical Resonator, arXiv:1302.6179.

[8] K. W. Murch, K. L. Moore, S. Gupta, and D. M. Stamper-Kurn, Observation of Quantum-Measurement
Backaction with an Ultracold Atomic Gas, Nat. Phys. 4, 561 (2008).

[9] T. P. Purdy, R. W. Peterson, and C. A. Regal, Observation of Radiation Pressure Shot Noise on a Macroscopic Object, Science 339, 801 (2013).

[10] T. A. Palomaki, J. W. Harlow, J. D. Teufel, R. W. Simmonds, and K. W. Lehnert, Coherent State Transfer between Itinerant Microwave Fields and a Mechanical Oscillator, Nature (London) 495, 210 (2013).

[11] T. A. Palomaki, J. D. Teufel, R. W. Simmonds, and K. W. Lehnert, Entangling Mechanical Motion with Microwave Fields, Science 342, 710 (2013).

[12] S. G. Hofer, W. Wieczorek, M. Aspelmeyer, and K. Hammerer, Quantum Entanglement and Teleportation in Pulsed Cavity Optomechanics, Phys. Rev. A 84, 052327 (2011).

[13] T. J. Kippenberg, H. Rokhsari, T. Carmon, A. Scherer, and K. J. Vahala, Analysis of Radiation-Pressure Induced Mechanical Oscillation of an Optical Microcavity, Phys. Rev. Lett. 95, 033901 (2005).

[14] T. Carmon, H. Rokhsari, L. Yang, T. J. Kippenberg, and K. J. Vahala, Temporal Behavior of Radiation-PressureInduced Vibrations of an Optical Microcavity Phonon Mode, Phys. Rev. Lett. 94, 223902 (2005).

[15] M. Ludwig, C. Neuenhahn, C. Metzger, A. Ortlieb, I. Favero, K. Karrai, and F. Marquardt, Self-Induced Oscillations in an Optomechanical System Driven by Bolometric Backaction, Phys. Rev. Lett. 101, 133903 (2008).

[16] G. Anetsberger, O. Arcizet, Q. P. Unterreithmeier, R. Rivière, A. Schliesser, E. M. Weig, J. P. Kotthaus, and T. J. Kippenberg, Near-Field Cavity Optomechanics with Nanomechanical Oscillators, Nat. Phys. 5, 909 (2009).

[17] I. S. Grudinin, H. Lee, O. Painter, and K. J. Vahala, Phonon Laser Action in a Tunable Two-Level System, Phys. Rev. Lett. 104, 083901 (2010).

[18] S. Zaitsev, A. K. Pandey, O. Shtempluck, and E. Buks, Forced and Self-Excited Oscillations of an Optomechanical Cavity, Phys. Rev. E 84, 046605 (2011).

[19] F. Marquardt, J. G. E. Harris, and S. M. Girvin, Dynamical Multistability Induced by Radiation Pressure in HighFinesse Micromechanical Optical Cavities, Phys. Rev. Lett. 96, 103901 (2006).

[20] S. Zaitsev, O. Gottlieb, and E. Buks, Nonlinear Dynamics of a Microelectromechanical Mirror in an Optical Resonance Cavity, Nonlinear Dynamics 69, 1589 (2012).

[21] J. B. Khurgin, M. W. Pruessner, T. H. Stievater, and W. S. Rabinovich, Laser-Rate-Equation Description of Optomechanical Oscillators, Phys. Rev. Lett. 108, 223904 (2012).

[22] J. B. Khurgin, M. W. Pruessner, T. H. Stievater, and W. S. Rabinovich, Optically Pumped Coherent Mechanical Oscillators: The Laser Rate Equation Theory and Experimental Verification, New J. Phys. 14, 105022 (2012).

[23] M. Ludwig, B. Kubala, and F. Marquardt, The Optomechanical Instability in the Quantum Regime, New J. Phys. 10, 095013 (2008).

[24] K. J. Vahala, Back-action Limit of Linewidth in an Optomechanical Oscillator, Phys. Rev. A 78, 023832 (2008).

[25] D. A. Rodrigues and A. D. Armour, Amplitude Noise Suppression in Cavity-Driven Oscillations of a Mechanical Resonator, Phys. Rev. Lett. 104, 053601 (2010). 
[26] A. D. Armour and D. A. Rodrigues, Quantum Dynamics of a Mechanical Resonator Driven by a Cavity, C.R. Phys. 13, 440 (2012).

[27] J. Qian, A. A. Clerk, K. Hammerer, and F. Marquardt, Quantum Signatures of the Optomechanical Instability, Phys. Rev. Lett. 109, 253601 (2012).

[28] P. D. Nation, Nonclassical Mechanical States in an Optomechanical Micromaser Analogue, Phys. Rev. A 88, 053828 (2013).

[29] M. Dykman and M. Krivoglatz, Spectral Distribution of Nonlinear Oscillators with Nonlinear Friction Due to a Medium, Phys. Status Solidi B 68, 111 (1975).

[30] M. Lax, Classical Noise. V. Noise in Self-Sustained Oscillators, Phys. Rev. 160, 290 (1967).

[31] F. Haake and M. Lewenstein, Adiabatic Expansion for the Single-Mode Laser, Phys. Rev. A 27, 1013 (1983).

[32] C. Gardiner and P. Zoller, Quantum Noise: A Handbook of Markovian and Non-Markovian Quantum Stochastic Methods with Applications to Quantum Optics, Springer Series in Synergetics (Springer, New York, 2004).

[33] J. B. Khurgin, Phonon Lasers Gain a Sound Foundation, Physics 3, 16 (2010).

[34] P. Rabl, Photon Blockade Effect in Optomechanical Systems, Phys. Rev. Lett. 107, 063601 (2011).

[35] A. Nunnenkamp, K. Børkje, and S. M. Girvin, Single-Photon Optomechanics, Phys. Rev. Lett. 107, 063602 (2011).

[36] I. Wilson-Rae, N. Nooshi, W. Zwerger, and T. J. Kippenberg, Theory of Ground State Cooling of a Mechanical Oscillator Using Dynamical Backaction, Phys. Rev. Lett. 99, 093901 (2007).

[37] F. Marquardt, J. P. Chen, A. A. Clerk, and S. M. Girvin, Quantum Theory of Cavity-Assisted Sideband Cooling of Mechanical Motion, Phys. Rev. Lett. 99, 093902 (2007).

[38] The zero-point amplitude is $\sqrt{\hbar / m \omega_{m}}$ for an oscillator of mass $m$.

[39] G. D. Mahan, Many-Particle Physics, Physics of Solids and Liquids (Springer, New York, 2000).

[40] P. D. Drummond and D.F. Walls, Quantum Theory of Optical Bistability. I. Nonlinear Polarisability Model, J. Phys. A 13, 725 (1980).

[41] A. Mari and J. Eisert, Gently Modulating Optomechanical Systems, Phys. Rev. Lett. 103, 213603 (2009).

[42] C. Genes, D. Vitali, P. Tombesi, S. Gigan, and M. Aspelmeyer, Ground-State Cooling of a Micromechanical Oscillator: Comparing Cold Damping and Cavity-Assisted Cooling Schemes, Phys. Rev. A 77, 033804 (2008).
[43] R. Ghobadi, A. R. Bahrampour, and C. Simon, Quantum Optomechanics in the Bistable Regime, Phys. Rev. A 84, 033846 (2011).

[44] S. Aldana, C. Bruder, and A. Nunnenkamp, On the Equivalence between an Optomechanical System and a Kerr Medium, Phys. Rev. A 88, 043826 (2013).

[45] G. Heinrich, M. Ludwig, J. Qian, B. Kubala, and F. Marquardt, Collective Dynamics in Optomechanical Arrays, Phys. Rev. Lett. 107, 043603 (2011).

[46] Yu. M. Golubev and I. V. Sokolov, Photon Antibunching in a Coherent Light Source and Suppression of the Photorecording Noise, J. Exp. Theor. Phys. 60, 234 (1984).

[47] Yu. M. Golubev, Excitation of the Active Medium of a Micromaser by Light from a Sub-Poissonian Optical Laser, J. Exp. Theor. Phys. 80, 212 (1995).

[48] J. McKeever, A. Boca, A. D. Boozer, J. R. Buck, and H. J. Kimble, Experimental Realization of a One-Atom Laser in the Regime of Strong Coupling, Nature (London) 425, 268 (2003).

[49] S. Ya. Kilin and A. B. Mikhalychev, Single-Atom Laser Generates Nonlinear Coherent States, Phys. Rev. A 85, 063817 (2012).

[50] J. Dalibard, Y. Castin, and K. Mølmer, Wave-Function Approach to Dissipative Processes in Quantum Optics, Phys. Rev. Lett. 68, 580 (1992).

[51] R. Dum, P. Zoller, and H. Ritsch, Monte Carlo Simulation of the Atomic Master Equation for Spontaneous Emission, Phys. Rev. A 45, 4879 (1992).

[52] K. Mølmer, Y. Castin, and J. Dalibard, Monte Carlo WaveFunction Method in Quantum Optics, J. Opt. Soc. Am. B 10, 524 (1993).

[53] J. R. Johansson, P. D. Nation, and F. Nori, QuTiP: An OpenSource Python Framework for the Dynamics of Open Quantum Systems, Comput. Phys. Commun. 183, 1760 (2012).

[54] J. R. Johansson, P. D. Nation, and F. Nori, QuTiP 2: A Python Framework for the Dynamics of Open Systems, Comput. Phys. Commun. 184, 1234 (2013).

[55] T. E. Oliphant, Python for Scientific Computing, Comput. Sci. Eng. 9, 10 (2007).

[56] S. M. Barnett and P. L. Knight, Dissipation in a Fundamental Model of Quantum Optical Resonance, Phys. Rev. A 33, 2444 (1986).

[57] J. I. Cirac, Interaction of a Two-Level Atom with a Cavity Mode in the Bad-Cavity Limit, Phys. Rev. A 46, 4354 (1992). 